\title{
Regularizing and self-avoidance effects of integral Menger curvature
}

\author{
Pawel Strzelecki, Marta Szumańska and Heiko von der Mosel
}

\begin{abstract}
We investigate geometric curvature energies on closed curves involving integral versions of the Menger curvature. In particular, we prove geometric variants of Morrey-Sobolev and Morrey-space imbedding theorems, which may be viewed as counterparts to respective results on one-dimensional sets in the context of harmonic analysis.
\end{abstract}

Mathematics Subject Classification (2010): 28A75 (primary); 53A04, 46E35 (secondary).

\section{Introduction}

Let $\gamma: \mathbb{S}^{1} \rightarrow \mathbb{R}^{3}$ be a continuous, closed and rectifiable curve, with an arclength parametrization $\Gamma: S_{L} \cong \mathbb{R} / L \mathbb{Z} \rightarrow \mathbb{R}^{3}$, defined on the circle of length $L$. For three different arclength parameters $s, t, \sigma \in S_{L}$ we look at the radius $R(\Gamma(s), \Gamma(t), \Gamma(\sigma))$ of the smallest circle containing the curve points $\Gamma(s), \Gamma(t)$, and $\Gamma(\sigma)$, which in general is simply the circumcircle of these points. Now we ask the question:

How much information about the shape of $\gamma$ and about its arclength parametrization $\Gamma$ can be extracted from suitable evaluations of $R$ on all triples of distinct curve points?

The answer is: quite a lot, that is, sufficient control on $R$ along $\gamma$ both allows us to control local curvature and gives us information about how the curve is embedded in the ambient space $\mathbb{R}^{3}$. In fact, the function $R$ gives rise to a whole range of what we call geometric curvature energies with regularizing and self-avoidance effects.

It was the idea of Gonzalez and Maddocks [14] to successively search for the smallest such radii while varying one or several of the three curve points. In this way, one obtains for fixed arclength parameters $s, t \in S_{L}$ the intermediate radius

$$
\varrho[\gamma](s, t):=\inf _{\sigma \in S_{L} \backslash\{s, t\}} R(\Gamma(s), \Gamma(t), \Gamma(\sigma)),
$$


or for fixed $s \in S_{L}$ the so-called global radius of curvature

$$
\rho_{G}[\gamma](s):=\inf _{t \in S_{L} \backslash\{s\}} \varrho[\gamma](s, t),
$$

and finally,

$$
\triangle[\gamma]:=\inf _{s \in S_{L}} \rho_{G}[\gamma](s) .
$$

Motivated by analytical and computational issues arising in the natural sciences such as microbiology, Gonzalez and Maddocks were on the search for an analytically tractable notion of thickness for curves that does not rely on additional smoothness assumptions as, e.g., the normal injectivity radius in differential geometry requires. Indeed, a positive thickness $\Delta[\gamma]$ leads to embedded curves with bounded curvature, which is reflected in the following theorem proved in [15, Lemma 2], [29, Theorem 1]:

$\Delta[\gamma]$ is positive if and only if the arclength parametrization is injective and of class $C^{1,1} \cong W^{2, \infty}$.

The latter means that $\Gamma$ is not only continuously differentiable but has also a Lipschitz continuous tangent, which is equivalent to having bounded second weak derivatives of $\Gamma$ in the sense of Sobolev. This result was the starting point for a number of variational applications involving curves or elastic rods with positive thickness and under topological constraints [15, 5, 13, 11, 12]. However, the thickness $\Delta[\cdot]$ depends not only highly nonlinearly but also in a nonsmooth fashion on the curve $\gamma$, a fact which turned out to be a challenge for regularity investigations $[30,31,4]$, and for numerical treatment $[1,6,7]$.

Replacing the minimization in (1.3) by an integration one obtains a relaxed variant of a geometric curvature energy, namely

$$
\mathscr{U}_{p}(\gamma):=\left(\int_{S_{L}} \frac{d s}{\rho_{G}[\gamma](s)^{p}}\right)^{1 / p}, \quad p \in[1, \infty],
$$

where we notice that $\mathscr{U}_{\infty}(\gamma)=1 / \Delta[\gamma]$. In [35, Theorem 1] we could prove the following generalization of the above mentioned characterization of embedded Sobolev curves:

$\mathscr{U}_{p}$ is finite if and only if the arclength parametrization $\Gamma$ is injective and of class $W^{2, p}$ for $p \in(1, \infty]$.

Moreover, quantitative estimates allow for variational applications in the class of embeddings involving topological constraints analogous to those treated in [15]. For instance, we could show the existence of $\mathscr{U}_{p}$-minimizing curves in given knot classes; see [35, Theorem 5].

Substituting the remaining minimizations in (1.2) and (1.1) by integrations (as already suggested in [14] and [2]) we establish in the present paper the following 
results which may be viewed as "geometric Morrey-Sobolev imbedding theorems". In fact, one may view the integrands as $p$-th powers of (a kind of) curvature, i.e. as counterparts of $\left|\Gamma^{\prime \prime}\right|^{p}$, and one should notice the dimension of the respective domain of integration.

Theorem 1.1 (Geometric Morrey-Sobolev imbedding in two dimensions). If the curve $\gamma: \mathbb{S}^{1} \rightarrow \mathbb{R}^{3}$ satisfies

$$
\mathscr{I}_{p}(\gamma):=\int_{S_{L}} \int_{S_{L}} \frac{d s d t}{\varrho[\gamma](s, t)^{p}}<\infty \text { for some } p \in(2, \infty],
$$

then the arclength parametrization $\Gamma: S_{L} \rightarrow \mathbb{R}^{3}$ is injective and of class $C^{1,1-\frac{2}{p}}\left(S_{L}, \mathbb{R}^{3}\right)$.

Theorem 1.2 (Geometric Morrey-Sobolev imbedding in three dimensions). If $\gamma: \mathbb{S}^{1} \rightarrow \mathbb{R}^{3}$ satisfies

$$
\mathscr{M}_{p}(\gamma):=\int_{S_{L}} \int_{S_{L}} \int_{S_{L}} \frac{d s d t d \sigma}{R^{p}(\Gamma(s), \Gamma(t), \Gamma(\sigma))}<\infty \text { for some } p \in(3, \infty],
$$

and if $\Gamma: S_{L} \rightarrow \mathbb{R}^{3}$ is a local homeomorphism, then:

(i) $\Gamma \in C^{1,1-\frac{3}{p}}\left(S_{L}, \mathbb{R}^{3}\right)$;

(ii) The image $\Gamma\left(S_{L}\right)$ is $C^{1}$-diffeomorphic to the circle, and the mapping $\Gamma: S_{L} \rightarrow$ $\Gamma\left(S_{L}\right)$ is a $k$-fold covering for $k=L / d$, where

$$
d:=\inf \left\{|t-s|: t, s \in S_{L}, t \neq s \text { and } \Gamma(t)=\Gamma(s)\right\}>0 .
$$

Moreover, if there exists, in addition, at least one simple point of $\Gamma$, then $\Gamma$ is injective.

The considerably more complicated statement of Theorem 1.2 reflects the fact that the pure integral geometric curvature energy $\mathscr{M}_{p}(\cdot)$ controls merely the image of $\gamma$, which can be only transferred to regularity statements about the arclength parametrization $\Gamma$ under the mild additional assumption of a local homeomorphism. The more restrictive energy $\mathscr{I}_{p}(\cdot)$ inflicts direct control over the arclength parametrization due to the one minimization procedure left in the definition of $\varrho[\gamma](\cdot, \cdot)$ in $(1.1)$. This distinction between $\mathscr{M}_{p}$ and $\mathscr{I}_{p}$ can be illustrated by choosing a "bad" arclength parametrization $\tilde{\Gamma}:[0,4 \pi] \rightarrow \mathbb{S}^{1}$ of the unit circle $\mathbb{S}^{1}$ oscillating back and forth about one image point: $\tilde{\Gamma}$ has a discontinuous tangent with countably many discontinuities, and $\mathscr{I}_{p}(\tilde{\Gamma})$ is infinite because of the double points ( $c f$. examples in Section 3.2). The energy $\mathscr{M}_{p}(\tilde{\Gamma})$, however, is finite, since the integrand is constant and $\tilde{\Gamma}$ has finite length; see Section 3 for the details.

Both Theorem 1.1 and Theorem 1.2 follow via Hölder's inequality from the following more general result using only a local squared curvature condition, which can be interpreted as a "geometric Morrey-space imbedding": 
Theorem 1.3 (Geometric Morrey-space imbeddings). Assume that there are constants $\beta \in(0,1], r_{0}>0$, and $M_{0} \geq 0$ such that $\gamma: \mathbb{S}^{1} \rightarrow \mathbb{R}^{3}$ satisfies one of the following two conditions (where we denote $\left.B_{r}(x):=(x-r, x+r) \subset S_{L}\right)$ :

$$
\begin{gathered}
\int_{B_{r}\left(\tau_{1}\right)} \int_{B_{r}\left(\tau_{2}\right)} \frac{d s d t}{\varrho[\gamma](s, t)^{2}} \leq M_{0} r^{2 \beta} \\
\text { for all } r \in\left(0, r_{0}\right], \text { and } \tau_{1}, \tau_{2} \in S_{L} ; \\
\int_{B_{r}\left(\tau_{1}\right)} \int_{B_{r}\left(\tau_{2}\right)} \int_{B_{r}\left(\tau_{3}\right)} \frac{d s d t d \sigma}{R^{2}(\Gamma(s), \Gamma(t), \Gamma(\sigma))} \leq M_{0} r^{1+2 \beta} \\
\quad \text { for all } r \in\left(0, r_{0}\right], \tau_{1}, \tau_{2}, \tau_{3} \in S_{L},
\end{gathered}
$$

and, in addition, the arclength parametrization $\Gamma: S_{L} \rightarrow \mathbb{R}^{3}$ is a local homeomorphism.

Then $\Gamma \in C^{1, \beta}\left(S_{L}, \mathbb{R}^{3}\right)$ and statement (ii) of Theorem 1.2 holds true as well.

The assumption that $\Gamma$ be a local homeomorphism is necessary to control the behaviour of the parametrization. Even without this assumption we can say more about the image $\Gamma\left(S_{L}\right)$.

Theorem 1.4. Assume that there are constants $\beta \in(0,1], r_{0}>0$, and $M_{0} \geq 0$ such that the curve $\gamma$ satisfies (1.9). Then $\Gamma\left(S_{L}\right)$ is an embedded 1-dimensional submanifold of $\mathbb{R}^{3}$ (possibly with boundary) of class $C^{1, \beta}$.

Theorems 1.2, 1.3, and 1.4 have interesting counterparts in the framework of harmonic analysis. Here, it was Melnikov's and Verdera's [24, 25] discovery that the geometric curvature energy $\mathscr{M}_{p}$ for $p=2$ - called the total Menger curvature $^{1}$ - evaluated on one-dimensional Borel sets in the complex plane served as a crucial quantity to characterize removable sets for bounded analytic functions; consult also the surveys $[22,23,27,37]$. It follows from the work of Jones, and David and Semmes [9] (see [27, Theorem 39]) in that context that Ahlfors regular one-dimensional Borel sets $E \subset \mathbb{C}$ with

$$
\iiint_{\left(E \cap B_{r}(\xi)\right)^{3}} \frac{d \mathscr{H}^{1}(x) d \mathscr{H}^{1}(y) d \mathscr{H}^{1}(z)}{R^{2}(x, y, z)} \leq M_{0} r \text { for all } r \in\left(0, r_{0}\right], \xi \in \mathbb{C},
$$

are uniformly rectifiable, i.e., are contained in the graph of a bi-Lipschitz map $f: \mathbb{R} \rightarrow \mathbb{C}$. This resembles our geometric Morrey-space imbedding, Theorem 1.3, and also Theorem 1.4, on the level of (generally disconnected) one-dimensional

${ }^{1}$ Coined after the Austrian mathematician Karl Menger who considered the circumcircle radius formula purely in terms of distances to generalize differential geometric concepts such as curvature to general metric spaces; see [26, 3]. 
sets. The Morrey-Sobolev imbedding, Theorem 1.2, on the other hand, can be compared to Léger's remarkable result [18] stating that one-dimensional Borel sets $E \subset \mathbb{R}^{n}$ with finite total Menger curvature $\mathscr{M}_{2}(E)<\infty$ are in fact 1-rectifiable in the sense of geometric measure theory. That is, such sets $E$ are essentially contained in a countable union of Lipschitz graphs ${ }^{2}$. These relations to deep results of harmonic analysis generate a set of new questions such as: What regularity does the arclength parametrization of curves with finite $\mathscr{M}_{p}$-energy possess if $p \in[2,3]$ ? Under what circumstances do one-dimensional Borel sets $E$ with $\mathscr{M}_{p}(E)<\infty$, $p>3$, enjoy higher regularity? What about higher-dimensional analogues? Let us point out that a generalization of thickness $\Delta[\cdot]$ to two-dimensional surfaces with arbitrary co-dimension was introduced and investigated in [33,34]. A generalization of total Menger curvature $\mathscr{M}_{2}(\cdot)$ to higher-dimensional sets was recently initiated in $[19,20]$. In an ongoing research project we are investigating geometric curvature energies $\mathscr{M}_{p}(\cdot)$ for hypersurfaces; see the upcoming paper [36].

The central tool to prove Theorem 1.3 will be a "Uniform cone flatness theorem", Theorem 2.3 stated in Section 2 and proven in Section 5. It says that a curve with a local curvature condition (1.9) is locally contained in arbitrarily narrow cones, which can be interpreted as a "geometric differentiability", since it implies the existence of a tangent line at every point of $\gamma$. This opens up the pathway to proving uniform control of local injectivity of $\Gamma$ (see Proposition 2.7 and Corollary 2.8) as well as to prove differentiability of $\Gamma$ everywhere with a uniform estimate on the Hölder norm of $\Gamma^{\prime}$; see Theorem 2.10. Moreover, one can use Theorem 2.3 to obtain compactness results for families of curves with uniformly bounded $\mathscr{M}_{p}$-energy for some $p>3$. This opens the door to variational applications on embedded curves with topological constraints; see Section 4.

Section 3 is devoted to the self-avoidance effects that the local curvature conditions (1.8) and (1.9) imply. Assuming (1.9) and using the uniform cone flatness and its consequences, we prove there that $\Gamma\left(S_{L}\right)$ is a 1-dimensional embedded topological manifold in $\mathbb{R}^{3}$ (Theorem 3.1) and the embedding has to be tame (cf. Remark 3.2). Under the additional assumption that $\Gamma$ is a local homeomorphism, two things can happen. Either $\Gamma$ has at least one simple point; then, we prove in Theorem 3.7 that $\Gamma$ is in fact injective. Otherwise, if there are no simple points of $\Gamma$, then $\Gamma$ is a covering map. We establish this fact in Theorem 3.8. Finally, we show that assumption (1.8) is stronger than (1.9) and implies that $\Gamma$ must be injective.

The more technical proof of the Uniform cone flatness theorem is carried out in Section 5, whereas Section 6 contains subtle measure-theoretic and iterative arguments to improve the Hölder exponent of the derivative $\Gamma^{\prime}$ to reach the full statement of Theorem 1.3. Combining higher smoothness of $\Gamma$ with the fact that $\Gamma\left(S_{L}\right)$ is a topological manifold leads quickly to the proof of Theorem 1.4. Finally, in Section 7 we show that it is possible to state and prove all the main results of the

${ }^{2}$ Generalizations of this result to metric spaces were proved by Hahlomaa [16,17]; see also recent work of Schul [28]. For Borel sets of fractal dimensions $p / 2,0<p<2$, we refer to the work of Lin and Mattila [21]. 
paper assuming only that $\int_{B_{r}} \int_{B_{r}} \int_{B_{r}} \frac{1}{R} \leq K_{0} r^{2+\beta}$ (which, by virtue of the Hölder inequality, is weaker than (1.9)).

ACKNOWLedgements. Special thanks go to Mariano Giaquinta who made it possible that the first and the third author could spend some time in the Spring of 2008 at the Centro di Ricerca E. de Giorgi at the Scuola Normale di Pisa. We also thank Joan Verdera, whom we met there, for several fruitful discussions about the relations to harmonic analysis. This research was supported by the DFG and MNiSW.

Last but not least, we are grateful to the anonymous referee of an earlier version of this paper. Due to her or his suggestion, our first proofs of Theorems 1.1 and 1.2 have been substantially reworked, which made it possible to include Theorem 1.3 in this paper.

\section{Uniform cone flatness and differentiability}

\subsection{Notation}

We introduce here some notation and additional conventions which will be used throughout the whole paper.

The curve and its parametrization(s). As already mentioned in the introduction, $\gamma: \mathbb{S}^{1} \rightarrow \mathbb{R}^{3}$ always is a continuous, closed and rectifiable curve; we use capitalization to denote its arclength parametrization $\Gamma: S_{L} \cong \mathbb{R} / L \mathbb{Z} \rightarrow \mathbb{R}^{3}$, defined on the circle $S_{L}$ of length $L$. We make no other a priori assumptions on $\gamma$.

To fix the terminology, we adopt the following:

Definition 2.1. We say $\Gamma: S_{L} \cong \mathbb{R} / L \mathbb{Z} \rightarrow \mathbb{R}^{3}$ is arclength iff $\Gamma$ is Lipschitz and $\left|\Gamma^{\prime}\right|=1$ almost everywhere on $S_{L}$.

Note that we do not require $\Gamma$ to be 1-1. Sometimes it will be convenient to slightly abuse the notation and to identify $\gamma$ with the image $\Gamma\left(S_{L}\right) \subset \mathbb{R}^{3}$. It will turn out later on that $\gamma=\Gamma\left(S_{L}\right)$ is a manifold so that one can always use another (injective) arclength parametrization of $\gamma$.

Cones, strips and diamonds. For $x \neq y \in \mathbb{R}^{3}$ and $\varepsilon \in\left(0, \frac{\pi}{2}\right)$ we denote by

$$
C_{\varepsilon}(x ; y):=\left\{z \in \mathbb{R}^{3}: \exists t \neq 0 \text { such that } \Varangle(t(z-x), y-x)<\frac{\varepsilon}{2}\right\}
$$

the double cone whose vertex is at the point $x$, with cone axis passing through $y$, and with opening angle $\varepsilon$.

For $a \neq b \in \mathbb{R}^{3}$ we introduce the open infinite "strip"

$$
U(a, b)=\left\{v \in \mathbb{R}^{3}:\langle v-a, b-a\rangle>0,\langle v-b, a-b\rangle>0\right\}
$$


and the closed half space

$$
H^{+}(a, b)=\left\{v \in \mathbb{R}^{3}:\langle v-a, b-a\rangle \geq 0\right\} .
$$

For technical reasons it is convenient to use cones with specific opening angles $\varepsilon$ depending on $a, b$ and the curve $\gamma$, given by

$$
\varepsilon(a, b, \gamma):=\left(c(\beta) M_{0}\right)^{\frac{1}{6-4 \beta}}|a-b|^{\frac{2 \beta}{6-4 \beta}} .
$$

Here, $\beta \in(0,1]$ and $c(\beta)$ denotes the constant from Theorem 2.3 (see (5.20) for its exact value). For this value of $\varepsilon$ we shall use the abbreviation

$$
D(a, b):=C_{\varepsilon(a, b, \gamma)}(a, b) \cap C_{\varepsilon(a, b, \gamma)}(b, a) .
$$

Finally, we set

$$
D_{U}(a, b):=D(a, b) \cap U(a, b),
$$

which is a diamond shaped region with tips $a$ and $b$ on the two parallel planes bounding the strip $U(a, b)$. We shall be using the sets (2.2) and (2.3) only for points $a, b \in \gamma$ that are sufficiently close to guarantee that $\varepsilon(a, b, \gamma)<\pi / 2$.

Lenses and doughnuts. For $a \neq b \in \mathbb{R}^{3}$ and $r>0$, we write

$$
\ell(a, b ; r):=\bigcap\left\{B_{r}: a, b \in \partial B_{r}\right\}
$$

to denote the "lens-shaped" region which is formed by the intersection of all open balls $B_{r}$ of radius $r$ that contain both points $a, b$ on their boundary $\partial B_{r}$. We also write

$$
V(a, b ; r):=\bigcup\left\{B_{r}: a, b \in \partial B_{r}\right\}
$$

to denote the "thick (degenerate) doughnut" formed by the union of all such balls.

Finally, let us note two simple facts which are used very often in the estimates.

Lemma 2.2 (Angle bounds). The following estimates hold:

(i) If $x, y \in D_{U}(a, b)$, then

$$
\left|\frac{x-a}{|x-a|}-\frac{y-a}{|y-a|}\right| \leq \varepsilon(a, b, \gamma) .
$$

(ii) If $x \in \ell(a, b ; r)$, then

$$
\Varangle\left(\frac{x-a}{|x-a|}, \frac{b-a}{|b-a|}\right) \leq \arcsin \frac{|a-b|}{2 r} .
$$




\subsection{Uniform cone flatness}

Numerous properties of classes of rectifiable curves $\gamma$ satisfying the local curvature condition (2.8) - like the differentiability of their arclength parametrizations $\Gamma$, Hölder continuity of $\Gamma^{\prime}$, but also the fact that under a mild additional assumption each such arclength parametrization $\Gamma$ must be either injective or a $k$-fold covering - are based on the following crucial result.

Theorem 2.3 (Uniform cone flatness). Assume that there are constants $\beta \in(0,1]$, $r_{0}>0$, and $M_{0} \geq 0$, such that

$$
\begin{aligned}
\int_{B_{r}\left(\tau_{1}\right)} \int_{B_{r}\left(\tau_{2}\right)} \int_{B_{r}\left(\tau_{3}\right)} \frac{d s d t d \sigma}{R^{2}(\Gamma(s), \Gamma(t), \Gamma(\sigma))} & \leq M_{0} r^{1+2 \beta} \\
\text { for all } 0<r & \leq r_{0}, \text { and } \tau_{1}, \tau_{2}, \tau_{3} \in S_{L} .
\end{aligned}
$$

Then there exists a constant $c=c(\beta)>1$ depending only on $\beta$ such that if two numbers, $\varepsilon \in(0, \pi / 2)$ and $\eta>0$, satisfy the relation

$$
\varepsilon^{6-4 \beta} \geq c(\beta) M_{0} \eta^{2 \beta} \text { and } \eta \leq \min \left\{\frac{1}{2} \operatorname{diam} \Gamma\left(S_{L}\right), r_{0}\right\},
$$

then for every $s, t \in S_{L}$ such that $|\Gamma(s)-\Gamma(t)|=\eta$ we have

$$
\Gamma\left(S_{L}\right) \cap B_{2 \eta}(\Gamma(s)) \subset C_{\varepsilon}(\Gamma(s) ; \Gamma(t)) .
$$

Remark 2.4. When

$$
\mathscr{M}_{p}(\gamma)=\int_{S_{L}} \int_{S_{L}} \int_{S_{L}} \frac{d x d y d z}{R^{p}(\Gamma(x), \Gamma(y), \Gamma(z))}<\infty
$$

for some $p>3$, then assumption (2.8) is satisfied with $\beta=1-\frac{3}{p}$ and $M_{0}=$ $2^{3\left(1-\frac{2}{p}\right)} \mathscr{M}_{p}^{\frac{2}{p}}(\gamma)$. This follows easily from the Hölder inequality for exponents $p / 2$ and $p /(p-2)$, since

$$
\begin{aligned}
& \int_{B_{r}\left(\tau_{1}\right)} \int_{B_{r}\left(\tau_{2}\right)} \int_{B_{r}\left(\tau_{3}\right)} \frac{d x d y d z}{R^{2}(\Gamma(x), \Gamma(y), \Gamma(z))} \\
& \leq(2 r)^{3\left(1-\frac{2}{p}\right)}\left(\int_{B_{r}\left(\tau_{1}\right)} \int_{B_{r}\left(\tau_{2}\right)} \int_{B_{r}\left(\tau_{3}\right)} \frac{d x d y d z}{R^{p}(\Gamma(x), \Gamma(y), \Gamma(z))}\right)^{2 / p}
\end{aligned}
$$

and $3\left(1-\frac{2}{p}\right)=1+2\left(1-\frac{3}{p}\right)$. Similarly, if $\mathscr{I}_{p}(\gamma)<\infty$ for some $p \in(2, \infty]$ then (2.8) holds true as well.

Remark 2.5. It seems that our technique of proof of the Uniform cone flatness theorem does not allow a weakening of condition (2.8) with respect to the centers $\tau_{1}, \tau_{2}$, and $\tau_{3}$. We do need three different centers for the balls in the domain of 
integration, in contrast to the corresponding result of Jones, David and Semmes mentioned in the introduction; see (1.10). However, once we have shown initial Hölder regularity of the tangent $\Gamma^{\prime}$ with some Hölder exponent $\alpha \in(0, \beta)$ we can improve the Hölder exponent up to $\beta$ using a growth condition weaker than (2.8), namely growth on balls with coinciding centers; see (6.2).

Note also that for $M_{0}=0$ the theorem is trivial since in that case $\gamma$ must be a segment of a straight line.

We postpone the proof of Theorem 2.3 for a while and discuss its consequences.

Since the statement of Theorem 2.3 is symmetric in the parameters $s$ and $t$, we immediately infer:

Corollary 2.6 (Diamond property I). Assuming (2.8) for $\beta \in(0,1], r_{0}>0$, and $M_{0} \geq 0$, we obtain for all $x, y \in S_{L}$ with

$$
\delta:=|\Gamma(x)-\Gamma(y)| \leq \min \left\{\operatorname{diam} \gamma / 2, r_{0},\left(c(\beta) M_{0}+1\right)^{-1 / 2 \beta}\left(\frac{\pi}{2}\right)^{\frac{6-4 \beta}{2 \beta}}\right\}
$$

the inclusion

$$
\Gamma\left(S_{L}\right) \cap B_{2 \delta}(\Gamma(x)) \subset D(\Gamma(x), \Gamma(y)),
$$

for

$$
\varepsilon=\left(c(\beta) M_{0}\right)^{\frac{1}{6-4 \beta}}|\Gamma(x)-\Gamma(y)|^{\frac{2 \beta}{6-4 \beta}} .
$$

In order to prove that $\Gamma^{\prime}$ exists everywhere, and to establish the global injectivity of $\Gamma$, we need to impose an extra assumption on $\Gamma$ ( $c f$. the examples in Section 3). The following statement shall be useful.

Proposition 2.7 (Uniform local injectivity I). Assume that (2.8) holds for some constants $\beta \in(0,1], r_{0}>0$, and $M_{0} \geq 0$, and suppose that an arclength parametrization $\Gamma$ of $\gamma$ is a local homeomorphism. There exists a $\delta_{0}=\delta_{0}\left(\beta, M_{0}\right)>0$ such that if a closed interval $I=\left[x_{1}, x_{2}\right] \subset S_{L}$ satisfies

$$
\operatorname{diam} \Gamma(I) \leq \delta_{0},
$$

then $\left.\Gamma\right|_{\left[x_{1}, x_{2}\right]}$ is injective.

Since the arclength parametrization is Lipschitz with constant 1, this proposition implies the following:

Corollary 2.8 (Uniform local injectivity II). If (2.8) holds for some constants $\beta \in$ $(0,1], r_{0}>0$, and $M_{0} \geq 0$, and if $\Gamma$ is a local homeomorphism, then $\Gamma$ is injective on each interval $I \subset S_{L}$ with $\mathscr{L}^{1}(I)=\delta_{0}\left(\beta, M_{0}\right)>0$. 
Proof of Proposition 2.7. Fix

$$
\delta_{0}=\delta_{0}\left(\beta, M_{0}\right):=\frac{1}{8} \min \left\{\operatorname{diam} \gamma, r_{0},\left(c(\beta) M_{0}+1\right)^{-1 / 2 \beta}\left(\frac{\pi}{4}\right)^{\frac{6-4 \beta}{2 \beta}}\right\},
$$

so that

$$
\varepsilon_{0}:=\left(c(\beta) M_{0}\right)^{\frac{1}{6-4 \beta}} \delta_{0}^{\frac{2 \beta}{6-4 \beta}}<\frac{\pi}{4} .
$$

Choose any $I=\left[x_{1}, x_{2}\right] \subset S_{L}$ with diam $\Gamma(I) \leq \delta_{0}$. Without loss of generality we suppose that $x_{1}=0 \in S_{L}$ and $\Gamma\left(x_{1}\right)=0 \in \mathbb{R}^{3}$. To establish injectivity of $\Gamma$ on $I$, we shall prove that $f(t):=|\Gamma(t)-\Gamma(0)|$ is strictly increasing on $\left[0, x_{2}\right]$.

Assume the contrary. Then $f$ must have a local maximum (not necessarily strict) at some $t_{0} \in\left(0, x_{2}\right)$. We can assume $f\left(t_{0}\right)>0$ since otherwise $f \equiv 0$ on an interval which is not possible since $\Gamma$ is a local homeomorphism. Note that $f\left(t_{0}\right) \leq \operatorname{diam} \Gamma(I) \leq \delta_{0}$.

In every neighbourhood of $t_{0}$ we can find $t_{1} \neq t_{2}$ such that

$$
\Gamma\left(t_{1}\right), \Gamma\left(t_{2}\right) \in \partial B_{\delta}(0) \cap H^{+}\left(0, \Gamma\left(t_{0}\right)\right),
$$

for some $0<\delta=\left|\Gamma\left(t_{i}\right)-\Gamma(0)\right| \leq f\left(t_{0}\right) \leq \delta_{0}(i=1,2)$. By Corollary 2.6, we have

$$
\Gamma\left(S_{L}\right) \cap B_{2 \delta}(0) \subset D\left(0, \Gamma\left(t_{1}\right)\right) .
$$

Recall from (2.2) that $D\left(0, \Gamma\left(t_{1}\right)\right)=C_{\varepsilon\left(0, \Gamma\left(t_{1}\right), \gamma\right)}\left(0, \Gamma\left(t_{1}\right)\right) \cap C_{\varepsilon\left(0, \Gamma\left(t_{1}\right), \gamma\right)}\left(\Gamma\left(t_{1}\right), 0\right)$ with $\varepsilon\left(0, \Gamma\left(t_{1}\right), \gamma\right) \leq \varepsilon_{0}$ defined in $(2.12)$.

Hence, again by Corollary 2.6,

$$
\begin{aligned}
\Gamma\left(t_{2}\right) & \in \Gamma\left(S_{L}\right) \cap \partial B_{\delta}(0) \cap H^{+}\left(0, \Gamma\left(t_{0}\right)\right) \\
& \subset D\left(0, \Gamma\left(t_{1}\right)\right) \cap \partial B_{\delta}(0) \cap H^{+}\left(0, \Gamma\left(t_{0}\right)\right) \\
& =\left\{\Gamma\left(t_{1}\right)\right\} .
\end{aligned}
$$

Thus in every neighbourhood of $t_{0}$ there exist different points $t_{1}, t_{2}$ such that $\Gamma\left(t_{1}\right)=$ $\Gamma\left(t_{2}\right)$. This contradicts the assumption that $\Gamma$ is a local homeomorphism.

Combining Corollary 2.6 and 2.8 we obtain another:

Corollary 2.9 (Diamond property II). Under the assumptions of Corollary 2.8, we have

$$
\Gamma((x, x+t)) \subset D_{U}(\Gamma(x), \Gamma(x+t))
$$

for each $x \in S_{L}$ and each $t \in\left(0, \delta_{0}\left(\beta, M_{0}\right)\right)$.

Now we are ready to prove the existence and continuity of the derivative of every arclength parametrization of any curve satisfying the local curvature condition (2.8). 
Theorem 2.10. Assume that (2.8) holds for some constants $\beta \in(0,1], r_{0}>0$, and $M_{0} \geq 0$, and that the arclength parametrization $\Gamma: S_{L} \rightarrow \mathbb{R}^{3}$ of the curve $\gamma: \mathbb{S}^{1} \rightarrow \mathbb{R}^{3}$ is a local homeomorphism. Then $\Gamma^{\prime}$ is defined everywhere on $S_{L}$, and there is a constant $C_{\Gamma}$ depending on diam $\gamma, r_{0}, \beta$, and $M_{0}$, such that

$$
\left|\Gamma^{\prime}(s)-\Gamma^{\prime}(t)\right| \leq C_{\Gamma}|s-t|^{\frac{2 \beta}{6-4 \beta}} \quad \text { for all } s, t \in S_{L}
$$

Moreover, for $|s-t| \leq \delta_{0}\left(\beta, M_{0}\right)$ inequality (2.13) holds with $C_{\Gamma}=\left(c(\beta) M_{0}\right)^{\frac{1}{6-4 \beta}}$.

Proof. First we chose $x \in S_{L}$ and $0<t<\delta_{0}=\delta_{0}\left(\beta, M_{0}\right)$ (where $\delta_{0}$ is the number from Proposition 2.7 and Corollary 2.8) such that $\Gamma^{\prime}(x), \Gamma^{\prime}(x+t)$ exist and have length 1. By Corollary 2.9 we have

$$
\Gamma((x, x+t)) \subset D_{U}(\Gamma(x), \Gamma(x+t)) .
$$

Hence, for any two points $x_{1}, x_{2}$ such that $x<x_{1}<x_{2}<x+t$ the difference quotients

$$
\frac{\Gamma\left(x_{1}\right)-\Gamma(x)}{x_{1}-x} \text { and } \quad \frac{\Gamma\left(x_{2}\right)-\Gamma(x+t)}{x_{2}-(x+t)}
$$

belong to the same one-sided cone with opening angle $\varepsilon(\Gamma(x), \Gamma(x+t), \gamma) \leq \varepsilon_{0}$ defined by (2.12). This observation implies that $\Gamma^{\prime}(x)$ and $\Gamma^{\prime}(x+t)$ (which are unit vectors!) differ at most by $\varepsilon(\Gamma(x), \Gamma(x+t), \gamma)$; see Lemma 2.2.

Therefore,

$$
\left|\Gamma^{\prime}(x)-\Gamma^{\prime}(y)\right| \leq\left(c(\beta) M_{0}\right)^{\frac{1}{6-4 \beta}}|x-y|^{\frac{2 \beta}{6-4 \beta}}
$$

for all $x, y \in S_{L}$ such that $|x-y| \leq \delta_{0}$ and $\Gamma^{\prime}(x), \Gamma^{\prime}(y)$ exist and have length 1 .

Next, a standard calculation shows that

$$
\left|\Gamma^{\prime}(x)-\Gamma^{\prime}(y)\right| \leq \tilde{C}\left(\beta, M_{0}\right)|x-y|^{\frac{2 \beta}{6-4 \beta}}
$$

also for pairs of parameters $x, y \in S_{L}$ with $|x-y|>\delta_{0}$, where the derivatives exist and have length 1 . Hence, $\Gamma^{\prime}$ has a unique Hölder continuous extension to the whole parameter circle $S_{L}$ satisfying the same Hölder estimate, i.e. (2.13) for

$$
C_{\Gamma}:=\max \left\{\left(c(\beta) M_{0}\right)^{\frac{1}{6-4 \beta}}, 2 \delta_{0}\left(\beta, M_{0}\right)^{-\frac{2 \beta}{6-4 \beta}}\right\}
$$

As $\Gamma$ is Lipschitz, we have $\Gamma(t)-\Gamma(s)=\int_{s}^{t} \Gamma^{\prime}(\tau) d \tau$ for all parameters $s, t \in S_{L}$. Therefore, it is a routine matter to check that this extension yields the derivative of $\Gamma$ at each point of $S_{L}$. 


\section{The image of $\Gamma$, self-avoidance, injectivity}

\subsection{The image of $\Gamma$ and its topological properties}

In this section we prove that every curve $\Gamma$ which satisfies the assumptions of the Uniform cone flatness theorem (Theorem 2.3) is an embedded topological submanifold of $\mathbb{R}^{3}$ and, if $\Gamma$ is injective in addition, it is tame ( $c f$. Remark 3.2 below). In particular, by Remark 2.4, simple curves with finite $\mathscr{M}_{p}$-energy, $p>3$, are tame. This fact is comparable with the corresponding result of Freedman, He, and Wang [10, Section 4] for the Möbius energy, only that their proof is more involved due to lack of regularity.

Theorem 3.1. Assume that $\Gamma: S_{L} \rightarrow \mathbb{R}^{3}$ satisfies the assumptions of Theorem 2.3. Then, $\gamma=\Gamma\left(S_{L}\right)$ is a 1-dimensional topological submanifold of $\mathbb{R}^{3}$ (possibly with boundary), i.e., $\gamma$ is homeomorphic to a circle or a closed segment.

Proof. It suffices to show that for each point $P \in \gamma$ there exists an open neighbourhood $\Omega \subset \mathbb{R}^{3}$ containing $P$ such that $\Omega \cap \gamma$ is homeomorphic to the open interval $(-1,1)$ where $0 \in(-1,1)$ corresponds to $P$, or such that $\Omega \cap \gamma$ is homeomorphic to the interval $[0,1)$ again with $0 \in[0,1)$ corresponding to the (boundary point) $P$. The proof of this fact is based on the Uniform Cone Flatness theorem and its immediate consequences.

We fix $P \in \gamma$. Let $\delta_{0} \equiv \delta_{0}\left(\beta, M_{0}\right)>0$ be defined by (2.11), and let $B:=$ $B_{\delta_{0}}(P)$.

Invoking connectivity of $\gamma$ and applying Corollary 2.6 for $\varepsilon=\pi / 4$, we see that there exists a point $Q \in \gamma \cap \partial B$ such that $Q$ is connected to $P$ by an $\operatorname{arc} \gamma_{P Q} \subset B$ of $\gamma$; we have

$$
\gamma \cap B \subset C_{\pi / 4}(P, Q) \cap C_{\pi / 4}(Q, P)
$$

and

$$
\gamma_{P Q} \subset H^{+}(P, Q) .
$$

For sake of brevity, let $H^{+}:=H^{+}(P, Q)$ and $H^{-}:=\mathbb{R}^{3} \backslash$ int $H^{+}$. Theorem 2.3 guarantees that

(i) For each $r \in\left(0, \delta_{0}\right)$, the intersection $\gamma \cap H^{+} \cap \partial B_{r}(P)$ contains precisely one point of $\gamma$.

(ii) For each $r \in\left(0, \delta_{0}\right)$, the intersection $\gamma \cap H^{-} \cap \partial B_{r}(P)$ contains at most one point of $\gamma$.

Indeed, since $r<\delta_{0},(2.9)$ is satisfied for $\eta:=r$ and $\varepsilon:=\pi / 4$. Suppose (say) that

$$
Q_{1}, Q_{2} \in \gamma \cap H^{+} \cap \partial B_{r}(P)
$$

then, by Corollary 2.6,

$$
\left\{Q_{2}\right\} \subset C_{\pi / 4}\left(P, Q_{1}\right) \cap C_{\pi / 4}\left(Q_{1}, P\right) \cap C_{\pi / 4}(P, Q) .
$$


Thus, we necessarily have $Q_{2}=Q_{1}$. Moreover, (i) holds since the arc $\gamma_{P Q}$ joins $P$ to $Q \in \partial B$ and is contained in $H^{+}$. Now, two cases may happen.

Case 1 . There exists a number $\delta \in\left(0, \delta_{0}\right]$ such that

$$
\gamma \cap H^{-} \cap \partial B_{r}(P) \neq \varnothing \quad \text { for each } r \in(0, \delta]
$$

Case 2. For every number $\delta \in\left(0, \delta_{0}\right]$ we have

$$
\gamma \cap H^{-} \cap \partial B_{r}(P)=\emptyset \quad \text { for some } r \in(0, \delta] .
$$

If Case 2 holds, then - since $\gamma$ is connected - we have in fact

$$
\gamma \cap H^{-} \cap \partial B_{r}(P)=\emptyset \quad \text { for each } r \in\left(0, r_{1}\right],
$$

where $r_{1}$ is some positive number in $\left(0, \delta_{0}\right)$.

It is now straightforward to check that if (3.1) holds, then $\gamma \cap B_{\delta}(P)$ is homeomorphic to the interval $(-\delta, \delta)$, and if (3.3) holds, then $\gamma \cap$ int $B_{r_{1}}(P)$ is homeomorphic to the interval $\left[0, r_{1}\right)$ and $P$ is one of the boundary points of $\gamma$. To verify the details, take the obvious mapping

$$
f(Q)= \begin{cases}r & \text { for } Q \in \partial B_{r}(P) \cap H^{+}, \\ -r & \text { for } Q \in \partial B_{r}(P) \cap H^{-} .\end{cases}
$$

It is clear that $f$ is well defined and continuous. If the inverse $f^{-1}=: g$ were discontinuous, one could obtain a sequence $r_{j} \rightarrow r$ such that all points $Q_{j}=g\left(r_{j}\right)$ were at least at some positive distance from $Q=g(r)$. A contradiction follows from Corollary 2.6 (Diamond property I): $Q$ would not belong to the diamonds with vertices at $Q_{j}$ and $P$ for $j$ sufficiently large, but this is impossible.

Remark 3.2. Recall that a simple closed curve $\Gamma: S_{L} \hookrightarrow \mathbb{R}^{3}$ is tame if the embedding $\Gamma: S_{L}=S_{L} \times\{0\} \hookrightarrow \mathbb{R}^{3}$ extends to an embedding of $S_{L} \times B_{r}^{2}(0) \approx$ $S_{L} \times \mathbb{R}^{2} \hookrightarrow \mathbb{R}^{3}$. Equivalently, the embedding $\Gamma: S_{L} \hookrightarrow \mathbb{R}^{3}$ is tame if an ambient isotopy deforms the curve into some polygonal (embedded) curve in $\mathbb{R}^{3}$. Let us note that every simple closed curve $\Gamma: S_{L} \hookrightarrow \mathbb{R}^{3}$ which satisfies (2.8) is tame since $\Gamma \in C^{1}$ according to Theorem 2.10. (See the book of Crowell and Fox, [8, pages 147-152] who prove that every simple arclength parametrized $C^{1}$ curve is ambient isotopic to a regular embedded polygon.) It is an easy exercise to give another simple proof of tameness of $\Gamma$ by constructing directly the desired extension of the embedding, where one can use the fact that $\Gamma$ is $C^{1}$ and, like [10] do, refer to a result of Bing stating that every locally tame arc is tame.

The assumption that $\Gamma$ be simple (or closed) is of course not a serious restriction here. It follows from Theorem 3.1 that each curve $\Gamma: S_{L} \rightarrow \mathbb{R}^{3}$ which satisfies (2.8) has an injective arclength parametrization, and for this new parametrization an argument based on [8] works again: the arc $\Gamma\left(S_{L}\right)$ is tame. 


\subsection{Examples of "bad" parametrizations}

It is clear that if $\Gamma: S_{L} \rightarrow \mathbb{R}^{3}$ is an arclength mapping, then (2.8) depends in fact only on the shape of the image of $\Gamma$. This is why without some extra assumption $\Gamma$ might be nondifferentiable at some points.

Let

$$
F(s)=(\cos s, \sin s, 0) \quad \text { for } s \in[0,2 \pi] .
$$

Example 3.3. If $\varphi(t)=\pi-|\pi-t|$ for $t \in[0,2 \pi]$, then $\Gamma:=F \circ \varphi: S_{2 \pi} \rightarrow \mathbb{R}^{3}$ is arclength. Obviously, $\mathscr{M}_{p}[\Gamma]$ is finite for every $p>0,(2.8)$ is satisfied for $\beta=1$, and $\gamma=\Gamma\left(S_{L}\right)$ is a smooth 1-dimensional manifold with boundary (simply: a doubly covered semicircle), but $\Gamma$ is not differentiable at $t=\pi$, since $\Gamma$ is not a local homeomorphism.

Example 3.4. Assume that $A \subset[0,4 \pi]$ is an $\mathscr{H}^{1}$-measurable subset such that $\mathscr{H}^{1}(A)=3 \pi$ and moreover

$$
\mathscr{H}^{1}(A \cap[0, x]) \geq \frac{x}{2} \quad \text { for all } x \in[0,4 \pi] .
$$

Set $g_{A}(x):=2 \chi_{A}(x)-1\left(\right.$ i.e. $g_{A} \equiv 1$ on $A$ and $g_{A} \equiv-1$ off $A$ ), and

$$
\Phi_{A}(t):=\int_{0}^{t} g_{A}(x) d x
$$

(see Figure 3.1; here, $A$ is simply a union of several disjoint intervals). It is easy to see that $\Phi_{A}$ maps $[0,4 \pi]$ onto $[0,2 \pi], \Phi_{A}(0)=0, \Phi_{A}(4 \pi)=2 \pi$ and $\Phi_{A}^{\prime}(t)= \pm 1$ for almost every $t \in[0,4 \pi]$. The map $\Gamma:=F \circ \Phi_{A}$ is arclength, $\gamma=\Gamma\left(S_{4 \pi}\right)$ is a circle, $\mathscr{M}_{p}[\Gamma]$ is finite for every $p>0$, and (2.8) holds with $\beta=1$. However, $\Gamma$ is not differentiable everywhere (again, the reason is that $\Gamma$ is not a local homeomorphism).

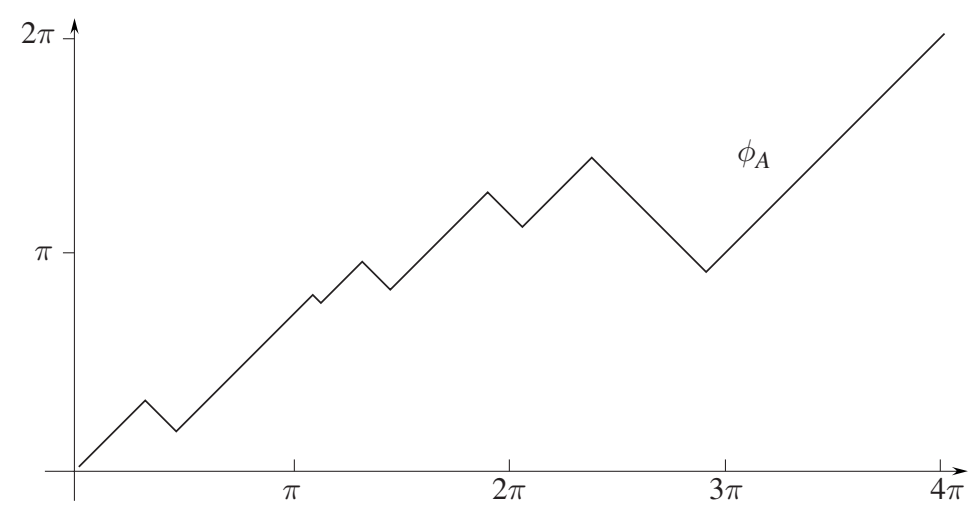

Figure 3.1. 


\subsection{Locally homeomorphic parametrizations}

A parameter $s \in S_{L}$ is called a simple point of $\Gamma$ if $\Gamma(t) \neq \Gamma(s)$ for every $t \in$ $S_{L}, t \neq s$. If $s \in S_{L}$ is not a simple point, we call it a multiple point; we say that $s \in S_{L}$ is a point of multiplicity $k$ if the cardinality of the set $\left\{t \in S_{L}: \Gamma(t)=\Gamma(s)\right\}$ is equal to $k$.

It is easy to see that if $\Gamma$ is a local homeomorphism, then all points $s \in S_{L}$ have finite multiplicity.

Lemma 3.5. If $\Gamma$ is a local homeomorphism then the set of all multiple points of $\Gamma$ is compact.

Proof. Since $S_{L}$ is compact, it is enough to show that the set of all multiple points of $\Gamma$ is closed.

Let $\left\{x_{n}\right\}_{n=1}^{\infty}$ be a convergent sequence of multiple points and let $x=\lim _{n \rightarrow \infty} x_{n}$. We will show that $x$ is also a multiple point.

For every $n$ there exists a point $y_{n} \neq x_{n}$ such that $\Gamma\left(x_{n}\right)=\Gamma\left(y_{n}\right)$. By continuity of $\Gamma$ we have

$$
\Gamma\left(x_{n}\right) \underset{n \rightarrow \infty}{\longrightarrow} \Gamma(x) \text { and } \Gamma\left(y_{n}\right) \underset{n \rightarrow \infty}{\longrightarrow} \Gamma(x) .
$$

Compactness of $S_{L}$ provides existence of a convergent subsequence $y_{n_{k}}$. Let $y=$ $\lim _{k \rightarrow \infty} y_{n_{k}}$; by continuity of $\Gamma$, we have $\Gamma\left(y_{n_{k}}\right) \rightarrow \Gamma(y)$. Of course $\Gamma(x)=\Gamma(y)$. In order to prove the lemma it suffices to show that $x \neq y$.

Suppose that $x=y$. Then sequences $x_{n_{k}}, y_{n_{k}}$ tend to $x$, thus for every $\delta>0$ we can find $k$ such that $x_{n_{k}}, y_{n_{k}} \in[x-\delta, x+\delta]$ and $\Gamma\left(x_{n_{k}}\right)=\Gamma\left(y_{n_{k}}\right)$. Therefore $\Gamma$ is not injective in any neighbourhood of $x$. That contradicts the assumption that $\Gamma$ is a local homeomorphism.

Lemma 3.6. Assume that (2.8) holds for some constants $\beta \in(0,1], r_{0}>0$, and $M_{0} \geq 0$, and suppose that $\Gamma$ is a local homeomorphism. If $0 \leq y<x<L$ are such that $|x-y|<\delta_{0}$, where $\delta_{0}$ is given by (2.11), then

$$
\Gamma\left(S_{L}\right) \cap D_{U}(\Gamma(x), \Gamma(y)) \subseteq \Gamma([y, x]) .
$$

Remark. This lemma simply means that locally, in every set $D_{U}(\Gamma(x), \Gamma(y))$, there are no points of $\gamma$ besides those that belong to the $\operatorname{arc} \Gamma([y, x])$.

Proof. Let $t \in S_{L} \backslash[y, x]$ be a point whose image belongs to $D_{U}(\Gamma(x), \Gamma(y))$. Set $\delta:=|\Gamma(x)-\Gamma(t)|$. Corollary 2.9 applied to $\Gamma((y, x))$ guarantees that

$$
A:=\Gamma([y, x]) \cap \partial B_{\delta}(\Gamma(x)) \cap D_{U}(\Gamma(x), \Gamma(y)) \neq \emptyset .
$$

Let $s \in[y, x]$ be a parameter such that $\Gamma(s)$ belongs to the set $A$ defined above. Then $|\Gamma(t)-\Gamma(x)|=|\Gamma(s)-\Gamma(x)|=\delta$ and Corollary 2.9 applied to $\Gamma((s, x))$ 
implies that

$$
\begin{aligned}
\Gamma(t) & \in \Gamma\left(S_{L}\right) \cap \partial B_{\delta}(\Gamma(x)) \cap D_{U}(\Gamma(y), \Gamma(x)) \\
& \subset \Gamma\left(S_{L}\right) \cap D_{U}(\Gamma(x), \Gamma(s)) \cap \partial B_{\delta}(\Gamma(x)) \\
& =\{\Gamma(s)\} .
\end{aligned}
$$

Thus $\Gamma(t)=\Gamma(s) \in \Gamma([y, x])$.

Theorem 3.7. Assume that (2.8) holds for some constants $\beta \in(0,1], r_{0}>0$, and $M_{0} \geq 0$, and suppose that the arclength parametrization $\Gamma: S_{L} \rightarrow \mathbb{R}^{3}$ of $\gamma: \mathbb{S}^{1} \rightarrow \mathbb{R}^{3}$ is a local homeomorphism. If there exists at least one simple point of $\Gamma$ then $\Gamma$ is injective.

Proof. Suppose that $\Gamma$ is not injective. Let $\tilde{x}$ be a given simple point; choose a multiple point $y$ which is nearest to $\tilde{x}$ (the existence of $y$ follows from Lemma 3.5). Without any loss of generality we can assume that $y, \tilde{x} \in[0, L), y<\tilde{x}, \Gamma(y)=0$, and the interval $(y, \tilde{x}]$ consists only of simple points of $\Gamma$. As $y$ is a multiple point, we have $\Gamma(y)=\Gamma(z)=0$ for some $z \neq y$.

We can select a simple point $x \in(y, \tilde{x}]$ such that

$$
|x-y|<\delta_{0}=\delta_{0}\left(\beta, M_{0}\right)
$$

with $\delta_{0}\left(\beta, M_{0}\right)$ as in (2.11). Set $r=|\Gamma(x)|$, then $r>0$ since $x$ is a simple point.

Let $\delta_{1}, \delta_{2}$ be the largest positive numbers such that $\Gamma\left(\left(z-\delta_{1}, z\right)\right), \Gamma((z, z+$ $\left.\left.\delta_{2}\right)\right) \subset B_{r}(0) \subset \mathbb{R}^{3}$. By Corollary 2.6,

$$
\Gamma\left(\left(z-\delta_{1}, z+\delta_{2}\right)\right) \subset D(0, \Gamma(x)) \cap B_{r}(0) .
$$

Moreover $\Gamma\left(\left(z-\delta_{1}, z+\delta_{2}\right)\right) \backslash\{0\}$ has empty intersection with $D_{U}(0, \Gamma(x))$. Indeed, Lemma 3.6 implies that

$$
\Gamma\left(\left(z-\delta_{1}, z+\delta_{2}\right)\right) \cap D_{U}(0, \Gamma(x)) \subset \Gamma([y, x]),
$$

but $(y, x]$ consists only of simple points and therefore its image under $\Gamma$ cannot contain any images of parameters that are close to $z$. Thus

$$
\Gamma\left(\left(z, z+\delta_{1}\right)\right) \subseteq \Gamma\left(S_{L}\right) \cap B_{r}(0) \cap H^{+}\left(0, \Gamma\left(z-\delta_{2}\right)\right)
$$

and by Corollary 2.6 we obtain

$$
\Gamma\left(\left(z, z+\delta_{1}\right)\right) \subseteq \Gamma\left(S_{L}\right) \cap D_{U}\left(\Gamma\left(z-\delta_{2}\right), 0\right) .
$$

Now Lemma 3.6 gives $\Gamma\left(\left[z, z+\delta_{1}\right]\right)=\Gamma\left(\left[z-\delta_{2}, z\right]\right)$. However this contradicts the assumption that $\Gamma$ is a local homeomorphism. 
Theorem 3.8. Assume that the curve $\gamma: \mathbb{S}^{1} \rightarrow \mathbb{R}^{3}$ satisfies (2.8) for some $\beta \in$ $(0,1], r_{0}$, and $M_{0} \geq 0$, and suppose that $\Gamma: S_{L} \rightarrow \mathbb{R}^{3}$ is a local homeomorphism and (locally) an arclength parametrization of $\gamma$ which has no simple points. Then the following statements are true:

(i) $\Gamma\left(S_{L}\right)$ is $C^{1}$-diffeomorphic to the circle;

(ii) $\Gamma$ is d-periodic on $S_{L}$, where

$$
d:=\inf \left\{|t-s|: t, s \in S_{L}, t \neq s \quad \text { and } \quad \Gamma(t)=\Gamma(s)\right\}>0 ;
$$

moreover, $\Gamma: S_{L} \rightarrow \Gamma\left(S_{L}\right)$ is a $k$-fold covering for $k=L / d$.

We know from Remark 2.4 that finite $\mathscr{M}_{p}$-energy of $\gamma$ for some $p>3$ implies the validity of assumption (2.8) in Theorem 3.8. Consequently the two statements above are true for any finite energy curve $\gamma$ with a locally homeomorphic arclength parametrization that has no simple points. In addition we can combine Fubini's theorem with the fact that $\Gamma$ is a $k$-fold, $d$-periodic covering to show

Corollary 3.9. Assume that $\mathscr{M}_{p}(\gamma)<\infty$ for some $p>3$ and that $\Gamma: S_{L} \rightarrow \mathbb{R}^{3}$ is a local homeomorphism and (locally) an arclength parametrization of $\gamma$ which has no simple points. Then

$$
\mathscr{M}_{p}\left[\Gamma, S_{L}\right]=k^{3} \mathscr{M}_{p}\left[\Gamma_{1}, S_{L_{1}}\right],
$$

where $L_{1}:=d=L / k$ and $\Gamma_{1}: S_{L_{1}} \rightarrow \mathbb{R}^{3}$ is defined by setting $\Gamma_{1}(x):=\Gamma(x)$ for $x \in\left[0, L_{1}\right)$.

Proof of Theorem 3.8. For clarity, the whole proof is divided into several steps.

Step 0. Preparation. Consider the number $d$ defined by (3.6). Hereafter, $d$ will be referred to as the minimal spacing (of multiple points of $\Gamma$ ).

It is an easy exercise to use compactness of $S_{L}$ and the fact that $\Gamma$ is a local homeomorphism to check that $d$ indeed is positive and moreover $d$ is achieved, i.e., there are two distinct points $x_{1}, x_{2} \in S_{L}$ such that

$$
x_{2}-x_{1}=\operatorname{dist}\left(x_{1}, x_{2}\right)=d, \quad \Gamma\left(x_{1}\right)=\Gamma\left(x_{2}\right)=P \in \mathbb{R}^{3} .
$$

Without loss of generality, composing $\Gamma$ with a rotation of $S_{L}$ and translation in the image if necessary, we assume that $P=0$ and

$$
\Gamma^{-1}(P)=\left\{x_{1}, x_{2}, \ldots, x_{k}\right\}, \quad \text { where } 0=x_{1}<x_{2}<\ldots<x_{k}<L .
$$

Our general aim will be to show that $L=k d$ and that $\Gamma$ is $d$-periodic on $S_{L}$. This readily implies the desired conclusion. 
Step 1. Two small arcs of $\Gamma$ to the right of $x_{1}$ and $x_{2}$ coincide. We choose $n>1$ such that

$$
r:=\frac{d}{n}<\frac{1}{2} \delta_{0} \equiv \frac{1}{2} \delta_{0}\left(\beta, M_{0}\right)
$$

with $\delta_{0}$ as in (2.11).

Let $\delta_{i}, \theta_{i}$ for $i \in\{1,2\}$ be the largest positive numbers satisfying

$$
\Gamma\left(\left(x_{i}-\theta_{i}, x_{i}+\delta_{i}\right)\right) \subset B_{r}(0) \quad \text { for } i \in\{1,2\} .
$$

Let $I_{j}=\left[x_{j}-\theta_{j}, x_{j}+\delta_{j}\right], j=1,2$. Corollary 2.6, Lemma 3.6, and the assumption that $\Gamma$ is a local homeomorphism imply

$$
\Gamma\left(I_{1}\right)=\Gamma\left(I_{2}\right) .
$$

Moreover, by Proposition 2.7, $\Gamma$ is injective on each of the intervals $I_{j}$, and one of the following possibilities holds: either

$$
\Gamma\left(\left(x_{1}-\theta_{1}, x_{1}\right)\right)=\Gamma\left(\left(x_{2}-\theta_{2}, x_{2}\right)\right) \text { and } \Gamma\left(\left(x_{1}, x_{1}+\delta_{1}\right)\right)=\Gamma\left(\left(x_{2}, x_{2}+\delta_{2}\right)\right)
$$

or

$$
\Gamma\left(\left(x_{1}-\theta_{1}, x_{1}\right)\right)=\Gamma\left(\left(x_{2}, x_{2}+\delta_{2}\right)\right) \text { and } \Gamma\left(\left(x_{1}, x_{1}+\delta_{1}\right)\right)=\Gamma\left(\left(x_{2}-\theta_{2}, x_{2}\right)\right) .
$$

But (3.11) cannot hold since it would imply that $\Gamma\left(x_{1}+\delta_{1}\right)=\Gamma\left(x_{2}-\theta_{2}\right)$ which is impossible since $d$ is the minimal spacing of multiple points. (It is easy to use injectivity of $\Gamma$ on $I_{j}$ to see that in fact we have $x_{1}<x_{1}+\delta_{1} \leq x_{2}-\theta_{2}<x_{2}$; the possibility $x_{1}+\delta_{1}<x_{2}-\theta_{2}$ contradicts the definition of $d$, whereas $x_{1}+\delta_{1}=x_{2}-\theta_{2}$ combined with the second equality of images in (3.11) shows that $\Gamma$ cannot be a homeomorphism in any neighbourhood of $x_{1}+\delta_{1}$.)

Thus, (3.10) must hold. Injectivity of $\Gamma$ on $I_{j}$ combined with the fact that $\Gamma$ is an arclength parametrization, gives now $\delta_{1}=\delta_{2}$.

Fix an arbitrary $t \in\left(0, \delta_{1}\right)$ and consider the point $\Gamma\left(x_{1}+t\right)$. It belongs to the image of $\left[x_{2}, x_{2}+\delta_{1}\right]$, thus we have $\Gamma\left(x_{1}+t\right)=\Gamma\left(x_{2}+t_{2}\right)$ for some $t_{2} \in\left(0, \delta_{1}\right)$. Since $\Gamma\left(x_{2}+\cdot\right)$ is injective on $\left(0, \delta_{1}\right)$ and $\left|\Gamma^{\prime}\right|=1$, we must have $t_{2}=t$ (the measures of two coinciding arcs are equal). claim.

Note now that we have $\delta_{1}=\delta_{2} \geq r$. Thus, we have proved the following

Claim 1. If $x_{1}<x_{2} \in S_{L}$ belong to the preimage of the same point in $\Gamma\left(S_{L}\right)$ and their distance $\left|x_{2}-x_{1}\right|$ is equal to the minimum spacing $d$, then

$$
\Gamma\left(x_{1}+t\right)=\Gamma\left(x_{2}+t\right) \quad \text { for all } t \in[0, r],
$$

where $r$ is given by (3.9).

We are now ready to move step by step along the parameter domain to reach the desired conclusion. 
Step 2. Induction, first part. We apply Claim 1 to $x_{1}+r$ and $x_{2}+r$ (this can be done since these are again two points in $S_{L}$ that are mapped by $\Gamma$ to the same point in $\mathbb{R}^{3}$ and their distance is equal to the minimum spacing $d$ ). This yields

$$
\Gamma\left(x_{1}+r+t\right)=\Gamma\left(x_{2}+r+t\right) \quad \text { for all } t \in[0, r] \text {. }
$$

Iterating this procedure finitely many times, we obtain

$$
\Gamma\left(x_{1}+t\right)=\Gamma\left(x_{2}+t\right) \quad \text { for all } t \in[0, d] .
$$

If $k=2$, we are done. Otherwise, since $x_{2}=x_{1}+d$, we see that $x_{2}+d$ is another point in $S_{L}$ with $\Gamma\left(x_{2}+d\right)=P=\Gamma\left(x_{3}\right)$. Minimality of $d$ gives now $x_{2}+d=x_{3}$.

Step 3. Induction, second part. We repeat the whole Step 1 and Step 2 with $x_{3}$ (respectively $x_{2}$ ) replacing $x_{2}$ (respectively $x_{1}$ ) to obtain the following:

$$
\Gamma\left(x_{1}+t\right)=\Gamma\left(x_{2}+t\right)=\Gamma\left(x_{3}+t\right) \quad \text { for all } t \in[0, d] .
$$

Easy finite induction yields $L=k d$,

$$
x_{j}=x_{1}+(j-1) d \quad \text { for all } j=1,2, \ldots, k,
$$

and

$$
\Gamma(t)=\Gamma(t+d) \quad \text { for all } t \in S_{L} .
$$

Obviously (again by minimality of $d$ ) $\Gamma$ is injective on each of the intervals $\left[x_{j}, x_{j}+\right.$ 1 ) and each of them is mapped to the whole image of $\Gamma$. This completes the proof of Theorem 3.8.

\subsection{Self-avoidance for intermediate energies}

In our earlier paper [32, Proposition 2.1] we have proved that closed curves $\gamma$ which satisfy $\mathscr{I}_{p}(\gamma)<\infty$ for some $p \geq 2$ have to be simple ( $c f$. (1.5) for the definition of $\mathscr{I}_{p}$ ). Here, for the sake of completeness, we prove a slightly stronger result which shall be used later, in Section 5, to prove Theorem 1.3 under Assumption (i).

Proposition 3.10. Assume that there are constants $\beta \in(0,1], r_{0}>0$ and $K_{0} \geq 0$ such that $\gamma: \mathbb{S}^{1} \rightarrow \mathbb{R}^{3}$ satisfies

$$
\int_{B_{r}\left(\tau_{1}\right)} \int_{B_{r}\left(\tau_{2}\right)} \frac{d s d t}{\varrho[\gamma](s, t)} \leq K_{0} r^{1+\beta} \quad \text { for all } r \in\left(0, r_{0}\right], \text { and } \tau_{1}, \tau_{2} \in S_{L} \text {. }
$$

Then $\gamma$ is simple, i.e., its arclength parametrization $\Gamma: S_{L} \rightarrow \mathbb{R}^{3}$ is injective.

Remark 3.11. It is easy to check that, by Schwarz inequality, (1.8) implies (3.13) with $K_{0}=2 M_{0}{ }^{1 / 2}$. If $\mathscr{I}_{p}(\gamma)<\infty$ for some $p>2$, then (3.13) with $\beta=1-\frac{2}{p}$ and $K_{0}=4^{(p-1) / p}\left(\mathscr{I}_{p}(\gamma)\right)^{1 / p}$ follows from Hölder inequality. 
Proof. We follow the proof from [32] and argue by contradiction. Assume that $\Gamma(0)=0=\Gamma\left(s_{1}\right)$ for some $s_{1} \in S_{L} \backslash\{0\}$. Let $\Gamma_{1}:=\Gamma\left(\left[0, s_{1}\right]\right)$ and $\Gamma_{2}:=$ $\Gamma\left(\left[s_{1}, L\right]\right)$. Since $\Gamma$ is the arclength parametrization of $\gamma$, it has no intervals of constancy. Thus,

$$
d:=\min \left(\operatorname{diam} \Gamma_{1}, \operatorname{diam} \Gamma_{2}\right)>0 .
$$

We consider the portion of $\gamma$ contained in $B_{d / 4}(0)$. Choose four parameters: $\sigma_{1}, \sigma_{2} \in$ $\left(0, s_{1}\right)$ and $t_{1}, t_{2} \in\left(s_{1}, L\right)$ such that

$$
\sigma_{1}<\sigma_{2}, \quad t_{1}<t_{2} \quad \text { and } \quad \Gamma\left(\sigma_{1}\right), \Gamma\left(\sigma_{2}\right), \Gamma\left(t_{1}\right), \Gamma\left(t_{2}\right) \in \partial B_{d / 4}(0)
$$

Now, fix a number $\varepsilon \in\left(0, \frac{d}{12}\right)$ which is smaller than the smallest gap between $0, \sigma_{1}, \sigma_{2}, s_{1}, t_{1}, t_{2}$, and $L$ in the natural ordering on $S_{L}$.

Take $s \in(0, \varepsilon) \subset S_{L}$. If $\Gamma(s)=0$, we set $A(s):=(0, s) \subset S_{L}$. In this case, since $\Gamma(s)=\Gamma(0)=0$, we have $\varrho(s, \sigma) \leq|\Gamma(\sigma)| / 2<s$ for all $\sigma \in A(s)$, and

$$
\frac{\mathscr{H}^{1}(A(s))}{\varrho[\gamma](s, \sigma)}>1 \quad \text { for all } \sigma \in A(s) .
$$

Next, suppose that $\Gamma(s) \neq 0$. Then we set

$$
A(s):=\left\{\sigma \in\left(s_{1}-\varepsilon, s_{1}+\varepsilon\right) \mid \Gamma^{\prime}(\sigma) \text { exists and } \Gamma(\sigma) \in B_{|\Gamma(s)|}(0)\right\} .
$$

For each $\sigma \in A(s)$ consider the open diameter ball $D B(s, \sigma)$ defined as follows:

$$
D B(s, \sigma):=B_{r}(a) \quad \text { for } \quad r:=\frac{|\Gamma(s)-\Gamma(\sigma)|}{2}, \quad a:=\frac{\Gamma(s)+\Gamma(\sigma)}{2} .
$$

Two cases are possible now.

Case 1. $\Gamma$ intersects $\partial D B(s, \sigma)$ transversally at $\Gamma(\sigma)$. Then we can find a parameter $t \in S_{L}, t$ close to $\sigma$, such that the point $\Gamma(t) \in D B(s, \sigma)$. Since $\Gamma\left(\sigma_{2}\right), \Gamma\left(t_{1}\right) \in$ $\partial B_{d / 4}(0)$ and since, by the choice of $\varepsilon$ and $\sigma$, we have

$$
D B(s, \sigma) \subset \subset B_{3 \varepsilon}(0) \subset \subset B_{d / 4}(0),
$$

there exists a parameter $\tau \in\left[\sigma_{2}, t_{1}\right]$ which is different from $\sigma$ and satisfies $\Gamma(\tau) \in$ $\partial D B(s, \sigma)$. Thus,

$$
\begin{aligned}
\varrho[\gamma](s, \sigma) & \leq R(\Gamma(s), \Gamma(\sigma), \Gamma(\tau))=\frac{|\Gamma(s)-\Gamma(\sigma)|}{2} \\
& \leq|\Gamma(s)| \quad \text { as } \Gamma(\sigma) \in B_{|\Gamma(s)|}(0) .
\end{aligned}
$$

(Notice that by definition of $R$ one has $\varrho[\gamma](s, \sigma) \geq|\Gamma(s)-\Gamma(\sigma)| / 2$ which together with (3.16) implies $\varrho[\gamma](s, \sigma)=|\Gamma(s)-\Gamma(\sigma)| / 2$ in this case.) 
Case 2. $\Gamma$ touches $\partial \mathrm{DB}(s, \sigma)$ at $\Gamma(\sigma)$, i.e. $\Gamma^{\prime}(\sigma) \perp(\Gamma(\sigma)-\Gamma(s))$. In this case, take a ball $B=B_{r^{\prime}}$ with radius $r^{\prime}$ slightly larger than that of $D B(s, \sigma)$ and such that $\Gamma(s), \Gamma(\sigma) \in \partial B_{r^{\prime}}$. Any such sphere $\partial B_{r^{\prime}}$ is intersected transversally by $\Gamma$ at $\Gamma(\sigma)$. Mimicking the reasoning for Case 1 , one checks that $\varrho[\gamma](s, \sigma) \leq r^{\prime}$. Taking the infimum over all $r^{\prime}>|\Gamma(s)-\Gamma(\sigma)| / 2$, we obtain

$$
\varrho[\gamma](s, \sigma) \leq|\Gamma(s)|
$$

also in this case.

Now, for each $s \in(0, \varepsilon) \subset S_{L}$ with $\Gamma(s) \neq 0$ we have

$$
\mathscr{H}^{1}(A(s)) \geq \min \{2|\Gamma(s)|, 2 \varepsilon\}=2|\Gamma(s)|>|\Gamma(s)|
$$

since $\Gamma$ is differentiable a.e. and $\Gamma\left(s_{1}\right)=0$. Thus, condition (3.14) holds also when $|\Gamma(s)| \neq 0$, i.e. when $A(s)$ is defined as in (3.15). Therefore,

$$
K_{0} \varepsilon^{1+\beta} \stackrel{(3.13)}{\geq} \int_{B_{\varepsilon}(0)} \int_{B_{\varepsilon}\left(s_{1}\right)} \frac{d \sigma d s}{\varrho[\gamma](s, \sigma)} \geq \int_{0}^{\varepsilon} \int_{A(s)} \frac{d \sigma d s}{\varrho[\gamma](s, \sigma)} \stackrel{(3.14)}{\geq} \int_{0}^{\varepsilon} 1 d s=\varepsilon
$$

This yields $K_{0} \varepsilon^{\beta} \geq 1$, a contradiction when $\varepsilon \in\left(0, \frac{d}{12}\right)$ is sufficiently small. The proof of Proposition 3.10 is complete now.

\section{Families of curves with equibounded energy}

In this section we show that families of simple closed curves which have equibounded $\mathscr{M}_{p}$-energy for some $p>3$ are compact in the $C^{1}$-topology. This is another consequence of Theorem 2.3. We begin with an explicit translation of this theorem to the setting of curves with finite $\mathscr{M}_{p}$-energy.

Corollary 4.1 (Uniform cone flatness II). Assume that $p>3$ and that $\Gamma: S_{L} \rightarrow$ $\mathbb{R}^{3}$ satisfies $\mathscr{M}_{p}(\gamma)<K$. There exists a constant $c=c(p, K)>1$ depending only on $p$ and $K$ such that if two numbers, $\varepsilon \in(0, \pi / 2)$ and $\eta>0$, satisfy the relation

$$
\varepsilon^{p+6} \geq c(p, K) \eta^{p-3} \text { and } \eta \leq \frac{1}{2} \operatorname{diam} \Gamma\left(S_{L}\right),
$$

then for every $s, t \in S_{L}$ such that $|\Gamma(s)-\Gamma(t)|=\eta$ we have

$$
\Gamma\left(S_{L}\right) \cap B_{2 \eta}(\Gamma(s)) \subset C_{\varepsilon}(\Gamma(s) ; \Gamma(t)) .
$$

Proof. It follows from the Hölder inequality, $c f$. also Remark 2.4, that a curve $\gamma$ with $\mathscr{M}_{p}(\gamma) \leq K$ satisfies the assumptions of Theorem 2.3 with $\beta=1-\frac{3}{p}$, $r_{0}=L / 2$ and

$$
M_{0}=2^{3\left(1-\frac{2}{p}\right)} \mathscr{M}_{p}(\gamma)^{2 / p} \leq 8(K+1) .
$$

Therefore, one can easily check that for $\beta=1-\frac{3}{p}$ inequality (2.9) in Theorem 2.3 is equivalent to (4.1). Corollary 4.1 follows. 
To prove that families of curves with equibounded energy are precompact in the $C^{1}$ topology, we need two auxiliary results. The first one ascertains that an upper bound for the $\mathscr{M}_{p}$-energy of a curve $\gamma$ implies a lower bound for the diameter of $\gamma$.

Lemma 4.2. Assume that $p>3$. For each $0<K<\infty$ there exists a constant $\Theta=\Theta[p, K]>0$, depending only on $p$ and $K$, such that whenever $\Gamma: S_{L} \rightarrow \mathbb{R}^{3}$ satisfies $\mathscr{M}_{p}[\Gamma] \leq K$, then we have

$$
\operatorname{diam} \Gamma\left(S_{L}\right) \geq \Theta[p, K] .
$$

Proof. Fix $\Gamma$ with $\mathscr{M}_{p}[\Gamma] \leq K$. Set $\varepsilon_{1}:=\frac{\pi}{8}$ and

$$
\Theta[p, K]:=2 \varepsilon_{1}^{(p+6) /(p-3)} c(p, K)^{1 /(3-p)} .
$$

From now on argue we by contradiction. Assume that diam $\Gamma\left(S_{L}\right)=: d<\Theta[p, K]$. Let $\eta_{1}:=d / 2$. Then $\varepsilon_{1}$ and $\eta_{1}$ satisfy

$$
\varepsilon_{1}^{p+6} \geq c(p, K) \eta_{1}^{p-3} \text { and } \eta_{1} \leq \frac{1}{2} \operatorname{diam} \Gamma\left(S_{L}\right),
$$

so that we are allowed to use (4.2) for appropriate pairs of points of the curve.

Now, fix two points $s, \sigma \in S_{L}$ such that $|\Gamma(s)-\Gamma(\sigma)|=d=\operatorname{diam} \Gamma\left(S_{L}\right)$. Obviously, $\Gamma\left(S_{L}\right)$ must be then contained in the closure of the strip $U:=U(\Gamma(s), \Gamma(\sigma))$. Pick $t \in S_{L}$ such that $\Gamma(t) \in U \cap \partial B_{\eta_{1}}(\Gamma(s))$ and use (4.2) for the points $\Gamma(s)$ and $\Gamma(t)$, replacing $\varepsilon, \eta$ by $\varepsilon_{1}, \eta_{1}$, to see that $\Gamma(\sigma)$ must belong to the closure of the cone $C_{\varepsilon_{1}}(\Gamma(s) ; \Gamma(t))$.

Thus, the angle between $\Gamma(t)-\Gamma(s)$ and $\Gamma(\sigma)-\Gamma(s)$ must be smaller than $\varepsilon_{1} / 2$. However, the tangent $\Gamma^{\prime}(s)$ is perpendicular to $\Gamma(\sigma)-\Gamma(s)$ since otherwise $\Gamma\left(S_{L}\right)$ would contain points at some positive distance from $U$, and its diameter would be too large. Hence, an arc of $\Gamma\left(S_{L}\right)$ close to $\Gamma(s)$ cannot be contained in the cone $C_{\varepsilon_{1}}(\Gamma(s) ; \Gamma(t))$, which is a contradiction to the Uniform cone flatness theorem, Corollary 4.1 .

Theorem 4.3. Assume that $p>3$ and $\Gamma: S_{L} \rightarrow \mathbb{R}^{3}$ is a simple curve with $\mathscr{M}_{p}(\gamma) \leq K$. There exists a constant $C_{0}=C_{0}(p, K)$, depending only on $p$ and $K$, such that

$$
\left|\Gamma^{\prime}(s)-\Gamma^{\prime}(t)\right| \leq C_{0}(p, K)|s-t|^{\alpha} \quad \text { for all } s, t \in S_{L},
$$

where $\alpha=(p-3) /(p+6) \in(0,1)$.

Proof. This follows from a careful inspection of the proof of Theorem 2.10. We know, $c f$. the proof of Corollary 4.1, that $\Gamma$ satisfies the assumptions of the Uniform cone flatness theorem with

$$
M_{0}:=8(K+1), \quad \beta:=1-\frac{3}{p}, \quad r_{0}:=L / 2 .
$$


As $\Gamma$ is $1-1$, Theorem 2.10 yields existence and Hölder continuity of $\Gamma^{\prime}$ : we have

$$
\left|\Gamma^{\prime}(s)-\Gamma^{\prime}(t)\right| \leq C_{\Gamma}|s-t|^{\alpha} \quad \text { for all } s, t \in S_{L},
$$

where $\alpha=\frac{2 \beta}{6-4 \beta}=(p-3) /(p+6)$ and the constant $C_{\Gamma}, c f .(2.15)$, is given by

$$
C_{\Gamma}:=\max \left\{\left(c(\beta) M_{0}\right)^{\frac{1}{6-4 \beta}}, 2 \delta_{0}\left(\beta, M_{0}\right)^{-\frac{2 \beta}{6-4 \beta}}\right\}
$$

with $\delta_{0}\left(\beta, M_{0}\right), c f .(2.11)$, defined as

$$
\delta_{0}=\delta_{0}\left(\beta, M_{0}\right):=\frac{1}{8} \min \left\{\operatorname{diam} \gamma, r_{0},\left(c(\beta) M_{0}+1\right)^{-1 / 2 \beta}\left(\frac{\pi}{4}\right)^{\frac{6-4 \beta}{2 \beta}}\right\} .
$$

Since $c(\beta)$, the constant from Theorem 2.3 , depends only on $\beta=1-\frac{3}{p}$, and $\operatorname{diam} \gamma \geq \Theta[p, K]>0$ by Lemma 4.2 , we have $\delta_{0} \geq d_{0}(p, K)$ for some positive constant $d_{0}(p, K)$ which depends only on $p$ and $K$. Thus, we have $C_{\Gamma} \leq C_{0}(p, K)$, where $C_{0}(p, K)$ depends only on $p$ and $K$.

Corollary 4.4. Assume that $K, L>0$ and $p>3$. Let $Q$ be a fixed point in $\mathbb{R}^{3}$. If a family of rectifiable simple closed curves $\gamma_{j}: S^{1} \rightarrow \mathbb{R}^{3}$ satisfies

$$
Q \in \gamma_{j}\left(S^{1}\right) \text { and } \mathscr{H}^{1}\left(\gamma_{j}\right)=L \text { forall } j, \text { and } \sup _{j=1,2, \ldots} \mathscr{M}_{p}\left(\gamma_{j}\right) \leq K \text {, }
$$

then there exists $v_{0}=v_{0}(p, K)>0$ such that the arclength parametrizations $\Gamma_{j}$ of $\gamma_{j}$ satisfy

$$
\left|\Gamma_{j}(s)-\Gamma_{j}(t)\right| \geq \min \left(v_{0}, \frac{|s-t|}{2}\right) \quad \text { for all } j \text { and all } s, t \in S_{L} .
$$

Moreover, the family of functions $\Gamma_{j}^{\prime}: S_{L} \rightarrow \mathbb{S}^{2} \subset \mathbb{R}^{3}$ is equicontinuous and $\left\{\Gamma_{j}\right\}$ contains a subsequence $\left\{\Gamma_{j_{k}}\right\}$ which for $j_{k} \rightarrow \infty$ converges in the $C^{1}$-topology to a simple arclength parametrized closed curve $\Gamma \in C^{1,(p-3) /(p+6)}\left(S_{L}, \mathbb{R}^{3}\right)$ with $Q \in \Gamma\left(S_{L}\right)$.

Proof. The proof is very similar to the proof of Corollary 3.3 in [32]; we provide the details for the sake of completeness. Since the $\gamma_{j}$ are simple, their arclength parametrizations are injective. The existence of a convergent subsequence of $\Gamma_{j}$ follows easily from Theorem 4.3 and the Arzela-Ascoli compactness theorem. Once (4.5) is established, injectivity of the limit curve $\Gamma$ follows from (4.5) upon passing to the limit $j_{k} \rightarrow \infty$.

Thus it is enough to prove (4.5). Consider $g_{j} \in C^{1}\left(S_{L} \times S_{L}\right)$ given by

$$
g_{j}(s, t):=\left|\Gamma_{j}(s)-\Gamma_{j}(t)\right|^{2} .
$$


Since the $\Gamma_{j}$ are uniformly bounded in $C^{1, \alpha}, \alpha=(p-3) /(p+6)$, it is easy to show that there is a constant $v_{1}=v_{1}(p, K)>0$ such that

$$
g_{j}(s, t) \geq \frac{|s-t|^{2}}{4} \quad \text { for all } j \text { and all } s, t \text { such that }|s-t| \leq v_{1}(p, K)
$$

see, e.g., Lemma 6.2 for details. Since $\Sigma=S_{L} \times S_{L} \backslash\left\{(s, t):|s-t|<v_{1}(p, K)\right\}$ is compact, we find for each $j$ a pair $\left(s_{j}, t_{j}\right) \in \Sigma$ such that

$$
g_{j}\left(s_{j}, t_{j}\right) \leq g_{j}(s, t) \quad \text { for all }(s, t) \in \Sigma \text {. }
$$

Now, we either have $\left|s_{j}-t_{j}\right|=v_{1}(p, K)$ in which case (4.6) implies

$$
g_{j}(s, t) \geq \frac{v_{1}(p, K)^{2}}{4} \quad \text { for all } s, t \in \Sigma
$$

or we have $\nabla g_{j}\left(s_{j}, t_{j}\right)=0$, which is equivalent to

$$
\Gamma_{j}^{\prime}\left(s_{j}\right) \perp\left(\Gamma_{j}\left(s_{j}\right)-\Gamma_{j}\left(t_{j}\right)\right) \quad \text { and } \quad \Gamma_{j}^{\prime}\left(t_{j}\right) \perp\left(\Gamma_{j}\left(s_{j}\right)-\Gamma_{j}\left(t_{j}\right)\right) \text {. }
$$

For each $j$, this implies that $\Gamma_{j}\left(S_{L}\right) \cap\left[B_{\eta_{j}}\left(\Gamma_{j}\left(s_{j}\right)\right) \cap B_{\eta_{j}}\left(\Gamma_{j}\left(t_{j}\right)\right)\right]$ is not contained in the intersection $C_{\pi / 4}\left(\Gamma_{j}\left(s_{j}\right) ; \Gamma_{j}\left(t_{j}\right)\right) \cap, C_{\pi / 4}\left(\Gamma_{j}\left(t_{j}\right) ; \Gamma_{j}\left(s_{j}\right)\right)$, where $\eta_{j}:=$ $\left|\Gamma_{j}\left(s_{j}\right)-\Gamma_{j}\left(t_{j}\right)\right|$. (The reason is that an arc of $\gamma_{j}$ is tangent to the line which is perpendicular to to the common axis of the two cones.) By virtue of Corollary 4.1 for $\varepsilon:=\pi / 4$ in combination with Lemma 4.2 this means, however, that

$$
\begin{aligned}
g_{j}(s, t) & \geq\left|\Gamma_{j}\left(s_{j}\right)-\Gamma_{j}\left(t_{j}\right)\right|^{2}=\eta_{j}^{2} \\
& >\min \left\{\frac{1}{4}\left(\operatorname{diam} \Gamma_{j}\left(S_{L}\right)\right)^{2},\left[\frac{(\pi / 4)^{\frac{p+6}{p-3}}}{(c(p, K))^{\frac{1}{p-3}}}\right]^{2}\right\} \\
& \geq \min \left\{\frac{1}{4} \Theta(p, K)^{2},\left[\frac{(\pi / 4)^{\frac{p+6}{p-3}}}{(c(p, K))^{\frac{1}{p-3}}}\right]^{2}\right\} \\
& =: v_{2}(p, K)>0 \quad \text { for all } j \in \mathbb{N},(s, t) \in \Sigma .
\end{aligned}
$$

Summarizing (4.9), (4.7), and (4.6), we obtain (4.5) with $v_{0}:=\min \left\{v_{1}(p, K) / 2\right.$, $\left.\sqrt{\nu_{2}(p, K)}\right\}$.

Remark 4.5. It turns out that in Corollary 4.4 we have $\mathscr{M}_{p}(\Gamma) \leq \lim \inf _{j_{k}} \mathscr{M}_{p}\left(\Gamma_{j_{k}}\right) \leq$ $K$. This follows from the continuity of $R(\cdot, \cdot, \cdot)$ at triples of pairwise distinct noncollinear points in $\mathbb{R}^{3}$ and from Fatou's lemma. 
Remark 4.6. As a possible variational application we mention a counterpart of [32, Theorem 3.4]: the minima of $\mathscr{M}_{p}$-energy are achieved in prescribed knot (or isotopy) classes. Set

$$
C_{L, k}:=\left\{\gamma \in C^{0}\left(S^{1}, \mathbb{R}^{3}\right): \text { length }(\gamma)=L, \gamma \text { isotopic to } k\right\},
$$

where $k$ is a given representative of a particular tame knot or isotopy class. Mimicking the proof from [32], one shows that $C_{L, k}$ contains an arclength parametrized curve $\Gamma \in C^{1, \alpha}\left(S_{L}, \mathbb{R}^{3}\right) \cap C_{L, k}, \alpha=(p-3) /(p+6)$, such that $\mathscr{M}_{p}(\Gamma)=$ $\inf _{C_{L, k}} \mathscr{M}_{p}(\cdot)$. (The key point is that Corollary 4.4 may be combined with the stability of isotopy classes under $C^{1}$ convergence.)

It is also clear that the inequality $\mathscr{M}_{p}(\gamma) \leq K$ might be used as a side condition for other one-dimensional variational problems for curves or rods (similar to those treated in [15] using the global curvature constraint $\Delta[\gamma]^{-1} \leq K$ instead), also when one needs to fix the knot class.

\section{Proof of the uniform cone flatness theorem}

This section is devoted to the proof of Theorem 2.3.

We may assume that $s=0$ and $\Gamma(0)=0$. Fix $0<\varepsilon<\pi / 2$ and $\eta>0$ satisfying (2.9), and set

$$
\eta_{N}:=\frac{\eta}{2^{N-1}} \quad \text { and } \quad r_{N}:=\frac{100 N^{2}}{\varepsilon} \eta_{N} \quad \text { for } N=1,2, \ldots
$$

Since $\eta \leq \operatorname{diam} \gamma / 2$ we can pick points $p_{N}=\Gamma\left(t_{N}\right) \in \partial B_{\eta_{N}}(0)$ where we set $t_{1}:=t$ so that $p_{1}=\Gamma(t)$. To investigate the location of $\Gamma\left(S_{L}\right)$ close to $0 \in \mathbb{R}^{3}$, for each of the points $p_{N}$ we consider three sets:

$$
\ell_{N}:=\ell\left(0, p_{N} ; r_{N}\right), \quad V_{N}:=V\left(0, p_{N} ; r_{N}\right),
$$

(cf. Subsection 2.1 for the definition of lenses and doughnuts), and finally

$$
K_{N}:=B_{2 \eta_{N}}(0) \backslash V_{N} .
$$

Before proceeding further, let us explain the rough idea of the proof. We will show first that for each $N=1,2, \ldots$ the portion of $\gamma$ contained in the ball $B_{2 \eta_{N}}(0)$ lies in the union of $K_{N}$ and $\ell_{N}$ with two small balls centered at 0 and $p_{N}$, respectively; thus, the situation depicted in Figure 1 below (where the curve bends too much between $p_{N}$ and 0) cannot happen. Otherwise, we could find three distinct subarcs $A_{1}, A_{2}, A_{3}$ of $\gamma$ such that every triangle with its three vertices on $A_{1}, A_{2}$, and $A_{3}$, has interior angles well separated from 0 and $\pi$. A tedious but in fact elementary computation shows these arcs would contribute too much to the energy, contradicting (2.8). 
Having confined the curve $\gamma$ to the narrow sets for each $N \in \mathbb{N}$ allows us to send $N$ to $\infty$ and to show that the curve indeed flattens at 0 ; this proves the desired result.

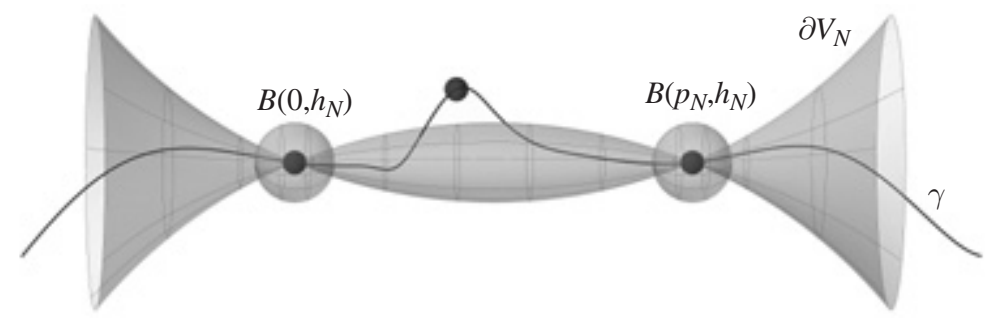

Figure 5.1. (This cannot happen). If $\gamma$ leaves the lens $\ell_{N}$ but contains some points of $V_{N} \backslash \ell_{N}$ sufficiently far away from 0 and $p_{N}$, then the local energy is too large, due to the behaviour of $R(\cdot, \cdot, \cdot)$ for triples of points that belong to the pieces of $\gamma$ contained in the interior of the three tiny dark balls.

To make all this precise let $\alpha_{N}$ denote the opening angle of the smallest cone with vertex at 0 containing the lenticular region $\ell_{N}$. In particular, one has

$$
\sin \frac{\alpha_{N}}{2}=\frac{\eta_{N}}{2 r_{N}},
$$

and moreover, we set

$$
\begin{aligned}
& h_{N}:=\operatorname{dist}\left(\frac{p_{N}+\Gamma(0)}{2}, \partial \ell_{N}\right)=r_{N}\left(1-\cos \frac{\alpha_{N}}{2}\right), \\
& \varphi_{N}:=\arctan \frac{2 h_{N}}{\eta_{N}}, \quad \tilde{h}_{N}:=h_{N} \sin \varphi_{N} .
\end{aligned}
$$

Finally, let $\delta_{N}$ be the smallest angle such that the cone $C_{\delta_{N}}\left(0 ; p_{N}\right)$ contains the union $\ell_{N} \cup K_{N}$, i.e. , $\delta_{N}=\alpha_{N}+2 \alpha_{N}^{\prime}$, where

$$
\alpha_{N}^{\prime}=\arcsin \frac{\eta_{N}}{r_{N}}=\arcsin \frac{\varepsilon}{100 N^{2}} .
$$

Thus, since $\operatorname{arc} \sin x \leq \pi x / 2$ on $[0,1]$, we have

$$
\delta_{N}=\alpha_{N}+2 \alpha_{N}^{\prime} \quad \text { and } \quad \sum_{N=1}^{\infty} \delta_{N} \leq \frac{3 \pi}{2} \sum_{N=1}^{\infty} \frac{\varepsilon}{100 N^{2}}<\frac{\varepsilon}{2} .
$$

(See also Figures 5.2-5.4 below.) 


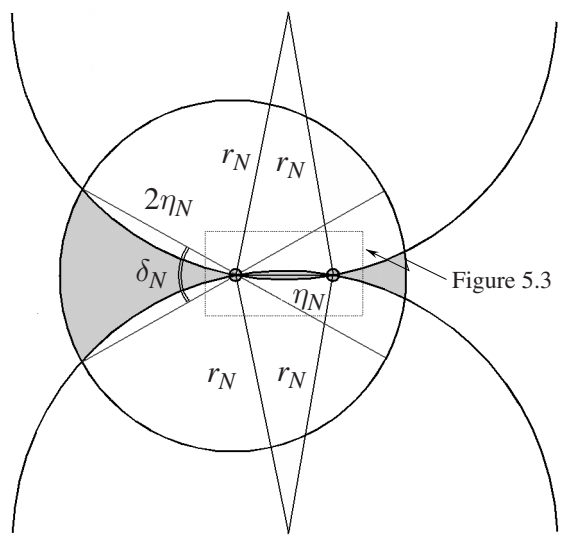

Figure 5.2. A plane passing through two points $0=\Gamma(0)$ and $p_{N}=\Gamma\left(t_{N}\right)$, located at the centers of two tiny shaded balls of radius $h_{N}$ in the middle part of the picture. Big arcs represent the boundaries of two of the balls of radius $r_{N}$ whose union is equal to the doughnut $V_{N}$. (Note: for $\varepsilon$ small and $N$ large, the ratio $r_{N} / \eta_{N}$ is in fact much larger than the figure shows.) Later on, see Claim 1 below and the details of its proof, we show that $\gamma \cap B_{2 \eta_{N}}(0)$ must in fact be contained in

$$
\ell_{N} \cup B_{h_{N}}\left(p_{N}\right) \cup B_{h_{N}}(0) \cup K_{N}
$$

that is, in that portion of the ball $B_{2 \eta_{N}}(0)$ which is formed by rotation of the shaded region in Figure 5.2 around the axis passing through 0 and $p_{N}$.
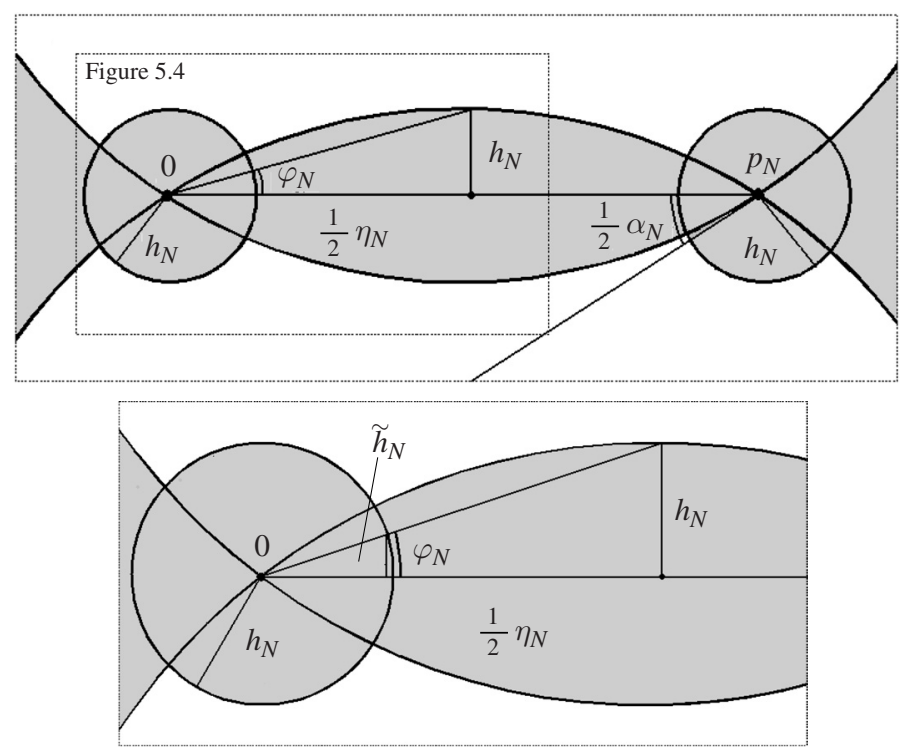

Figures 5.3 and 5.4. Enlarged parts of Figure 5.2, showing the location of 0 and $p_{N}$, the distances $\eta_{N}, h_{N}, \tilde{h}_{N}$, and the angles $\alpha_{N}, \varphi_{N}$ that are defined by (5.4)-(5.6). 
The main tool in the proof of Theorem 2.3 is the following:

Claim 5.1. For each $N=1,2, \ldots$ the following is true:

$$
\gamma \cap B_{2 \eta_{N}} \subset C_{\delta_{N}}\left(0 ; p_{N}\right) \cup B_{h_{N}},
$$

where the two balls $B_{2 \eta_{N}}$ and $B_{h_{N}}$ are centered at 0 .

(Later on, applying (5.8) iteratively, one can easily conclude the whole proof of Theorem 2.3.)

To prove Claim 5.1, we need the following elementary relations between the distances $h_{N}, \tilde{h}_{N}, \eta_{N}$ and $r_{N}$, which can be easily derived from Taylor expansions of trigonometric functions; see [32, Lemma 3.6] for details.

Lemma 5.2 (Relations between distances on Figures 5.2-5.4). For all $N=1,2, \ldots$ we have

$$
\begin{aligned}
\frac{\eta_{N}^{2}}{4 \pi r_{N}} & \leq h_{N} \leq \frac{\eta_{N}^{2}}{3 r_{N}}, \\
\frac{h_{N}^{2}}{\eta_{N}} & \leq \tilde{h}_{N} \leq \frac{2 h_{N}^{2}}{\eta_{N}}, \\
\frac{h_{N}}{\eta_{N}} & \geq \frac{\varepsilon}{400 \pi N^{2}} .
\end{aligned}
$$

The following two lemmas provide the key-estimates that will allow us to infer a large contribution to the energy once there is a point not contained in the shaded narrow zone depicted in Figure 5.2.

Lemma 5.3 (Estimate of $R(\cdot, \cdot, \cdot)$ when $\Gamma$ bends a lot). Fix $N=1,2, \ldots$ and suppose that for some $\tau_{0} \in S_{L}$ the point $\Gamma\left(\tau_{0}\right) \in B_{2 \eta_{N}}(0)$ but

$$
\Gamma\left(\tau_{0}\right) \notin \ell_{N} \cup B_{h_{N}}\left(p_{N}\right) \cup B_{h_{N}}(0) \cup K_{N}
$$

Then for all parameters $s \in \tilde{A}_{1}$ and $\sigma \in \tilde{A}_{2}$, where

$$
\begin{aligned}
& \tilde{A}_{1}=\left\{s \in S_{L}: \Gamma(s) \in B_{\tilde{h}_{N} / 10}\left(p_{N}\right)\right\} \\
& \tilde{A}_{2}=\left\{\sigma \in S_{L}: \Gamma(\sigma) \in B_{\tilde{h}_{N} / 10}(0)\right\}
\end{aligned}
$$

we have

$$
R\left(\Gamma(s), \Gamma(\sigma), \Gamma\left(\tau_{0}\right)\right) \leq 4 r_{N} .
$$

In fact, we simply have $R\left(q_{1}, q_{2}, \Gamma\left(\tau_{0}\right)\right) \leq 4 r_{N}$ whenever $q_{1} \in B_{\tilde{h}_{N} / 10}\left(p_{N}\right)$ and $q_{2} \in B_{\tilde{h}_{N} / 10}(0)$. 
Proof. If $\tau_{0}$ satisfies the assumptions of Lemma 5.3, then there exists a unique point $q \in \mathbb{R}^{3}$ determined by the following three conditions

(i) $\left|q-p_{N}\right|=|q|=r_{N}$,

(ii) $\Gamma\left(\tau_{0}\right) \in B_{r_{N}}(q) \backslash \ell_{N}$,

(iii) the four points $\Gamma\left(\tau_{0}\right), q, p_{N}$ and 0 are co-planar.

By elementary geometry,

$$
\frac{\alpha_{N}}{2} \leq \beta_{0}:=\Varangle\left(p_{N}-\Gamma\left(\tau_{0}\right), \Gamma(0)-\Gamma\left(\tau_{0}\right)\right) \leq \pi-\frac{\alpha_{N}}{2} .
$$

(This is easy to see: draw two circles $c_{1}, c_{2}$ of radius $r_{N}$, containing $p_{N}$ and $0=$ $\Gamma(0)$ and lying in the plane determined by $p_{N}, 0$ and $\Gamma\left(\tau_{0}\right)$. Then, $\beta_{0}=\pi-\alpha_{N} / 2$ when $\Gamma\left(\tau_{0}\right)$ lies on the short arc of $c_{1}$ connecting $p_{N}$ to 0 , and $\beta_{0}=\alpha_{N} / 2$ when $\Gamma\left(\tau_{0}\right)$ lies on the long arc of $c_{1}$ connecting $p_{N}$ to 0 . When $\Gamma\left(\tau_{0}\right)$ is between these two arcs, $\beta_{0}$ takes some intermediate value.)

For $s \in \tilde{A}_{1}$ and $\sigma \in \tilde{A}_{2}$, let $\beta(s, \sigma)$ denote the angle at $\Gamma\left(\tau_{0}\right)$ in the triangle with vertices $\Gamma(s), \Gamma(\sigma)$ and $\Gamma\left(\tau_{0}\right)$. We then have

$$
\left|\beta(s, \sigma)-\beta_{0}\right| \leq \beta_{1}+\beta_{2},
$$

where

$\beta_{1}:=\Varangle\left(p_{N}-\Gamma\left(\tau_{0}\right), \Gamma(s)-\Gamma\left(\tau_{0}\right)\right), \quad \beta_{2}:=\Varangle\left(\Gamma(0)-\Gamma\left(\tau_{0}\right), \Gamma(\sigma)-\Gamma\left(\tau_{0}\right)\right)$.

Since the distances of $\Gamma\left(\tau_{0}\right)$ to $p_{N}$ and to $0=\Gamma(0)$ exceed $h_{N}$, and because $s \in \tilde{A}_{1}$ and $\sigma \in \tilde{A}_{2}$, we have

$$
\beta_{i} \leq \beta_{\max }, \quad i=1,2,
$$

where $\sin \beta_{\max }=\tilde{h}_{N} / 10 h_{N}$. Hence, using the basic estimate $2 x / \pi \leq \sin x \leq x$,

$$
\beta_{\max } \leq \frac{\pi}{2} \sin \beta_{\max }=\frac{\pi \tilde{h}_{N}}{20 h_{N}} \stackrel{(5.10)}{\leq} \frac{\pi h_{N}}{10 \eta_{N}} \stackrel{(5.9)}{\leq} \frac{\pi \eta_{N}}{30 r_{N}} \stackrel{(5.4)}{=} \frac{\pi}{15} \sin \frac{\alpha_{N}}{2}<\frac{\alpha_{N}}{8} .
$$

Therefore, by (5.13) and (5.14), we obtain $\frac{\alpha_{N}}{4} \leq \beta(s, \sigma) \leq \pi-\frac{\alpha_{N}}{4}$ and

$$
\sin \beta(s, \sigma) \geq \sin \frac{\alpha_{N}}{4}
$$

Thus,

$$
\begin{aligned}
R\left(\Gamma(s), \Gamma(\sigma), \Gamma\left(\tau_{0}\right)\right) & =\frac{|\Gamma(s)-\Gamma(\sigma)|}{2 \sin \beta(s, \sigma)} \\
& \leq \frac{2 \eta_{N}}{2 \sin \left(\alpha_{N} / 4\right)} \\
& =\frac{2 \eta_{N}}{\sin \left(\alpha_{N} / 2\right)} \cos \frac{\alpha_{N}}{4} \leq \frac{2 \eta_{N}}{\sin \left(\alpha_{N} / 2\right)} \stackrel{(5.4)}{=} 4 r_{N}
\end{aligned}
$$


Lemma 5.4. If $\tau_{0} \in S_{L}$ satisfies the assumptions of Lemma 5.3, then

$$
R(\Gamma(s), \Gamma(\sigma), \Gamma(\tau)) \leq 4 r_{N}
$$

for all parameters

$$
\begin{aligned}
s \in A_{1}: & =\left\{s \in S_{L}: \Gamma(s) \in B_{\tilde{h}_{N} / 20}\left(p_{N}\right)\right\}, \\
\sigma \in A_{2}: & =\left\{\sigma \in S_{L}: \Gamma(\sigma) \in B_{\tilde{h}_{N} / 20}(0)\right\}, \\
\tau \in A_{3}: & =\left\{\tau \in S_{L}: \Gamma(\tau) \in B_{\tilde{h}_{N} / 20}\left(\Gamma\left(\tau_{0}\right)\right)\right\} .
\end{aligned}
$$

Proof. Note that for $s \in A_{1}, \sigma \in A_{2}$ and $\tau \in A_{3}$ we can shift the triangle with vertices $\Gamma(s), \Gamma(\sigma)$ and $\Gamma(\tau)$ by $\Gamma\left(\tau_{0}\right)-\Gamma(\tau)$, to obtain an adjacent triangle congruent to the original one with vertices $q_{1}, q_{2}$ and $\Gamma\left(\tau_{0}\right)$, where

$$
q_{1} \in B_{\tilde{h}_{N} / 10}\left(p_{N}\right) \text { and } q_{2} \in B_{\tilde{h}_{N} / 10}(0)
$$

by the triangle inequality. Now, we just invoke Lemma 5.3 to obtain

$$
R(\Gamma(s), \Gamma(\sigma), \Gamma(\tau))=R\left(q_{1}, q_{2}, \Gamma\left(\tau_{0}\right)\right) \leq 4 r_{N} .
$$

We are now ready for the crucial local energy estimate which proves Claim 1 by contradiction. Assume that Claim 1 were false. Fix $N \in \mathbb{N}$. Since

$$
C_{\delta_{N}}\left(0 ; p_{N}\right) \cup B_{h_{N}}(0) \supset \ell_{N} \cup B_{h_{N}}\left(p_{N}\right) \cup B_{h_{N}}(0) \cup K_{N},
$$

we would then find a parameter $\tau_{0}$ satisfying the assumptions of Lemma 5.4. Moreover, Lemma 5.2 implies

$$
\begin{aligned}
\tilde{h}_{N} & \leq \frac{2 h_{N}^{2}}{{ }_{(5.10)}} \leq \frac{2 \eta_{N}^{4}}{9 \eta_{N} r_{N}^{2}}=\frac{2}{9} \cdot \frac{\varepsilon^{2} \eta_{N}}{100^{2} N^{4}} \\
& <\frac{2}{3} \eta_{N} \leq \frac{2}{3} r_{0} .
\end{aligned}
$$

Since by definition each $A_{i}, i=1,2,3$, contains a ball $B_{i} \subset S_{L}$ of radius $\rho_{N}:=$ $\tilde{h}_{N} / 20$, we may apply Assumption (2.8) to estimate

$$
\begin{aligned}
\frac{h_{N}^{2+4 \beta}}{\left(10 \eta_{N}\right)^{1+2 \beta}} \underset{(5.10)}{\geq}\left(\frac{\tilde{h}_{N}}{20}\right)^{1+2 \beta}=\rho_{N}^{1+2 \beta} \underset{(2.8)}{\geq} \frac{1}{M_{0}} \int_{B_{1}} \int_{B_{2}} \int_{B_{3}} \frac{d \tau d \sigma d s}{R^{2}(\Gamma(s), \Gamma(\sigma), \Gamma(\tau))} \\
\underset{(5.16)}{\geq} \frac{1}{M_{0}} \cdot\left(4 r_{N}\right)^{-2} \mathscr{H}^{1}\left(B_{1}\right) \mathscr{H}^{1}\left(B_{2}\right) \mathscr{H}^{1}\left(B_{3}\right) \\
\quad=\frac{\left(2 \rho_{N}\right)^{3}}{4^{2} M_{0} r_{N}^{2}} \\
\underset{(5.10)}{\geq} \frac{1}{2 M_{0} r_{N}^{2}} \cdot \frac{h_{N}^{6}}{20^{3} \eta_{N}^{3}} \underset{(5.9)}{\geq} \frac{h_{N}^{8}}{2 \cdot 10^{3} M_{0} \eta_{N}^{7}} .
\end{aligned}
$$


This implies

$$
\begin{aligned}
& 200 M_{0} \geq \frac{h_{N}^{6-4 \beta}}{\eta_{N}^{6-2 \beta}}=\left(\frac{h_{N}}{\eta_{N}}\right)^{6-2 \beta} \cdot \frac{1}{h_{N}^{2 \beta}} \\
& \underset{(5.9)}{\geq} \frac{1}{(4 \pi)^{6-2 \beta}}\left(\frac{\eta_{N}}{r_{N}}\right)^{6-2 \beta} 3^{2 \beta}\left(\frac{r_{N}}{\eta_{N}^{2}}\right)^{2 \beta} \\
& \underset{(5.1)}{=} \frac{3^{2 \beta}}{(4 \pi)^{6-2 \beta}}\left(\frac{\varepsilon}{100 N^{2}}\right)^{6-2 \beta}\left(\frac{100 N^{2}}{\varepsilon \eta_{N}}\right)^{2 \beta} \\
&=\frac{300^{2 \beta}}{(5.1)} \cdot \varepsilon^{6-4 \beta} N^{8 \beta-12}\left(\frac{2^{N-1}}{\eta}\right)^{2 \beta},
\end{aligned}
$$

or

$$
200 M_{0} \cdot \frac{(400 \pi)^{6-2 \beta}}{300^{2 \beta}} \cdot \frac{N^{12-8 \beta}}{2^{2 \beta(N-1)}} \geq \frac{\varepsilon^{6-4 \beta}}{\eta^{2 \beta}}
$$

Thus, for

$$
c(\beta):=400^{7} \pi^{6} \cdot \max _{N \in \mathbb{N}} \frac{N^{12}}{2^{2 \beta(N-1)}} \geq 1
$$

we have

$$
\begin{aligned}
\frac{1}{2} c(\beta) M_{0} & =200 M_{0} \cdot(400 \pi)^{6} \cdot \max _{N \in \mathbb{N}} \frac{N^{12}}{2^{2 \beta(N-1)}} \\
& \geq 200 M_{0} \cdot \frac{(400 \pi)^{6-2 \beta}}{300^{2 \beta}} \cdot \max _{N \in \mathbb{N}} \frac{N^{12-8 \beta}}{2^{2 \beta(N-1)}} \quad \text { as } \beta \in(0,1] \\
& \stackrel{(5.19)}{\geq} \frac{\varepsilon^{6-4 \beta}}{\eta^{2 \beta}},
\end{aligned}
$$

so that by Assumption (2.9) we obtain the desired contradiction

$$
\frac{1}{2} c(\beta) M_{0} \geq \frac{\varepsilon^{6-4 \beta}}{\eta^{2 \beta}} \geq c(\beta) M_{0} .
$$

(Since $\eta, \varepsilon>0$, the first inequality in (5.21) implies that $M_{0}>0$; note that (2.8) holds with $M_{0}=0$ iff $R \equiv \infty$ for all triple of distinct nearby points of $\gamma$, i.e. when $\gamma$ is a piece of a straight line - and then Theorem 2.3 is obvious, anyway.)

Proof of Theorem 2.3 continued. Noting that $h_{N}<\eta_{N}$ and applying Claim 5.1 inductively, we obtain

$$
\Gamma\left(S_{L}\right) \cap B_{2 \eta}(0) \subset C_{\delta_{1}+\delta_{2} / 2+\cdots+\delta_{N} / 2}\left(0 ; p_{1}\right) \cup B_{h_{N}}(0), \quad N=2,3, \ldots
$$

since the axis of each of the successive cones lies in the preceding cone in the iteration. 
As the series $\sum \delta_{N}$ converges and its sum is smaller than $\varepsilon$ by (5.7), this yields

$$
\Gamma\left(S_{L}\right) \cap B_{2 \eta}(0) \subset C_{\varepsilon}\left(0 ; p_{1}\right) \cup B_{h_{N}}(0), \quad N=2,3, \ldots
$$

Since $h_{N} \rightarrow 0$ for $N \rightarrow \infty$, the intersection of all the sets $C_{\varepsilon}\left(0 ; p_{1}\right) \cup B_{h_{N}}(0)$ is equal to the cone $C_{\varepsilon}\left(0, p_{1}\right)$. This completes the whole proof of Theorem 2.3.

\section{From the local curvature conditions to higher regularity of $\Gamma^{\prime}$}

In this section we prove that rectifiable curves with local Menger energy decaying at least as fast as $r^{1+2 \beta}$ are of class $C^{1, \beta}$.

From now on, we assume that $\Gamma \in \operatorname{Lip}\left([0, L], \mathbb{R}^{3}\right)$ is an injective arclength parametrization of a rectifiable curve $\gamma$ in $\mathbb{R}^{3}$ satisfying

$$
\left|\Gamma^{\prime}(s)-\Gamma^{\prime}(t)\right| \leq C_{\Gamma}|t-s|^{\alpha} \quad \text { for all } s, t \in[0, L]
$$

for some $\alpha<\beta$.

(Note that if $\Gamma$ is an arclength parametrization and the decay assumption (2.8) is satisfied, then $\Gamma\left(S_{L}\right)$ is in fact, up to a homeomorphism, a circle or a segment. We simply reparametrize the curve in a 1-1 way; (2.8) is obviously still satisfied and, due to the results of Section 2, the new parametrization - possibly defined on a shorter interval, if the former $\Gamma$ was badly chosen - is of class $C^{1, \alpha}$ for $\alpha=$ $2 \beta /(6-4 \beta) \leq \beta$. It does not really matter in this Section whether $\Gamma(0)=\Gamma(L)$ or not.)

Instead of the local curvature condition (2.8) where one integrates over equally sized balls with three different centers we are going to use the following weaker condition $^{3}$ : there exists an $r_{0}>0$ and a constant $M_{0}$ such that

$$
\sup _{\substack{0<r \leq r_{0} \\ \tau \in S_{L}}} r^{-(1+2 \beta)} \int_{B_{r}(\tau)} \int_{B_{r}(\tau)} \int_{B_{r}(\tau)} \frac{d x d y d z}{R^{2}(\Gamma(x), \Gamma(y), \Gamma(z))} \leq M_{0} .
$$

Our aim will be to show that whenever $\Gamma$ satisfies both (6.1) and (6.2), then $\Gamma^{\prime}$ is of class $C^{\beta}\left(S_{L}, \mathbb{R}^{3}\right)$.

To carry out the proof of this fact, we introduce some notation. For an interval $J \subset S_{L}$, we write

$$
\underset{J}{\operatorname{osc}} \Gamma^{\prime}:=\max _{x, y \in J}\left|\Gamma^{\prime}(x)-\Gamma^{\prime}(y)\right|
$$

and set

$$
\Phi(t):=\sup _{\substack{J \subset S_{L} \\ \mathscr{H}^{1}(J) \leq t}}\left(\operatorname{osc} \Gamma^{\prime}\right) \quad \text { for } t \in[0, L] .
$$

The whole reasoning rests on the following key lemma.

3 Our method of proof for the Uniform cone flatness theorem, Theorem 2.3 (see Section 5 for the proof) does not seem to work under this weaker condition, we really do need three different centers in (2.8), compare with Remark 2.5. 
Lemma 6.1. Assume that (6.1) and (6.2) hold. Then there exist two numbers, $\delta_{1}=$ $\delta_{1}\left(M_{0}, C_{\Gamma}, \alpha, \beta\right)>0$, and $N=N(\alpha)>4$ with $6 N^{-\alpha}<\frac{1}{2}$, such that we have

$$
\left|\Gamma^{\prime}(u)-\Gamma^{\prime}(v)\right| \leq 6 \Phi\left(\frac{|u-v|}{N}\right)+c_{0}|u-v|^{\beta}
$$

whenever $u, v \in[0, L]$ and $|u-v| \leq \delta_{1}$. One can choose here $c_{0}=\operatorname{const}(\alpha) \cdot M_{0}^{1 / 2}$.

Remark. It is easy to note that this lemma implies $\Gamma^{\prime} \in C^{\beta}$. Indeed, taking the supremum of both sides of (6.4) with respect to $t \in\left[|u-v|, \delta_{1}\right]$, leads to

$$
\Phi(t) \leq 6 \Phi(t / N)+c_{0} t^{\beta} \quad \text { for } t \in\left[0, \delta_{1}\right] .
$$

Iterating this inequality and using $6 N^{-\beta}<6 N^{-\alpha}<1 / 2$, we easily obtain

$$
\Phi(t) \leq 6^{k} \Phi\left(t / N^{k}\right)+c_{0} t^{\beta} \sum_{j=0}^{\infty}\left(6 N^{-\beta}\right)^{j} \leq 6^{k} \Phi\left(t / N^{k}\right)+2 c_{0} t^{\beta}
$$

for each $k=1,2, \ldots$. Since we have assumed earlier that $\Gamma^{\prime} \in C^{\alpha}$, we have $\Phi(t) \leq C_{\Gamma} t^{\alpha}$, and $6 N^{-\alpha}<\frac{1}{2}$ by Lemma 6.1. This yields

$$
\Phi(t) \leq 6^{k} C_{\Gamma} \frac{t^{\alpha}}{N^{k \alpha}}+2 c_{0} t^{\beta} \leq C_{\Gamma} \frac{t^{\alpha}}{2^{k}}+2 c_{0} t^{\beta}
$$

for each $k=1,2, \ldots$ Upon passing to the limit $k \rightarrow \infty$, we conclude that

$$
\Phi(t) \leq 2 c_{0} t^{\beta} \quad \text { for all } t \in\left[0, \delta_{1}\right] .
$$

The rest of this section is divided into two parts. First, we prove Lemma 6.1. Next, we show how to assemble the proofs of all the theorems stated in the introduction, using Lemma 6.1 and the results of Sections 2-3.

\subsection{Proof of Lemma 6.1}

We begin with a simple observation.

Lemma 6.2. Assume that

$$
\left|\Gamma^{\prime}(t)-\Gamma^{\prime}(s)\right| \leq C_{\Gamma}|t-s|^{\alpha}, \quad t, s \in S_{L} .
$$

Then for every $\lambda \in(0,1)$ there exists $\delta_{2}=\delta_{2}\left(\lambda, C_{\Gamma}, \alpha\right)>0$ such that

$$
|\Gamma(t)-\Gamma(s)| \geq \lambda|t-s|
$$

for all $t, s \in S_{L}$ with $|t-s|<\delta_{2}$. 
Proof. Fix $s \in S_{L}$ and without loss of generality assume that $\Gamma^{\prime}(s)=(1,0,0)$. We then have

$$
\Gamma_{1}^{\prime}(t) \geq \Gamma_{1}^{\prime}(s)-\left|\Gamma_{1}^{\prime}(s)-\Gamma_{1}^{\prime}(t)\right| \geq 1-C_{\Gamma}|t-s|^{\alpha} \geq \lambda
$$

for all $t$ satisfying

$$
|t-s|<\delta_{2}:=\left(\frac{1-\lambda}{C_{\Gamma}}\right)^{1 / \alpha}
$$

Thus, for such $t$,

$$
|\Gamma(t)-\Gamma(s)| \geq\left|\Gamma_{1}(t)-\Gamma_{1}(s)\right|=\left|\int_{s}^{t} \Gamma_{1}^{\prime}(\tau) d \tau\right| \geq \lambda|t-s|,
$$

which completes the proof of Lemma 6.2.

We fix $N=N(\alpha)>4$ such that $6 N^{-\alpha}<\frac{1}{2}$. Next, we choose several other constants as follows:

$$
\begin{gathered}
\lambda \equiv \lambda(N):=1-\frac{1}{N^{2}} \\
c_{1}:=\frac{1}{3 N}\left(\frac{1}{2}-\frac{2}{N}\right) \in(0,1), \quad c_{2}:=\frac{1}{2}\left(\frac{c_{1}^{5}}{100 M_{0}}\right)^{1 / 2}, \\
\delta_{1}:=\min \left(\delta_{2}, c_{2}^{1 / \beta}, 1\right)>0,
\end{gathered}
$$

where $\delta_{2}$ is the constant from Lemma 6.2. Fix $u, v \in S_{L}, u<v,|u-v| \leq \delta_{1}$. Note that, by Lemma 6.2 and the definition of $\delta_{1}$, we have

$$
|\Gamma(s)-\Gamma(t)| \geq \lambda|s-t| \quad \text { for all } s, t \in[u, v] .
$$

Roughly speaking, the strategy of the proof is to find other parameters $x, y \in[u, v]$ which are very close to the endpoints of that interval and, due to energy bounds, are chosen so that $\left|\Gamma^{\prime}(x)-\Gamma^{\prime}(y)\right|$ is much smaller than one would presume knowing only that $\Gamma^{\prime} \in C^{\alpha}$.

Let $x, y \in[u, v]$ and let

$$
I_{x, y}:= \begin{cases}{[x, y]} & \text { if } x<y \\ {[y, x]} & \text { otherwise. }\end{cases}
$$

We define the following sets of bad parameters:

$$
\begin{aligned}
Z_{x, y}: & =\left\{z \in I_{x, y}: R(\Gamma(x), \Gamma(y), \Gamma(z)) \leq c_{2}|\Gamma(x)-\Gamma(y)|^{1-\beta}\right\}, \\
Y_{x}: & =\left\{y \in[u, v]: \mathscr{H}^{1}\left(Z_{x, y}\right) \geq c_{1}|\Gamma(x)-\Gamma(y)|\right\} \\
X: & =\left\{x \in[u, v]: \mathscr{H}^{1}\left(Y_{x}\right) \geq c_{1}|u-v|\right\} .
\end{aligned}
$$


Now, we estimate the measure of $X$ using Assumption (6.2) on Menger curvature of $\Gamma$ and two inequalities,

$$
\begin{array}{ll}
|\Gamma(x)-\Gamma(y)|^{-1} \geq|x-y|^{-1} \geq|u-v|^{-1} & \text { for all } x, y \in[u, v], \\
|\Gamma(x)-\Gamma(y)|^{2 \beta} \geq \lambda^{2 \beta}|x-y|^{2 \beta} \geq \frac{1}{4}|x-y|^{2 \beta} & \text { for all } x, y \in[u, v] .
\end{array}
$$

(The second one follows from (6.8), as we have $\lambda \in(1 / 2,1)$ and $2 \beta \leq 2$.) Using condition (6.2), we write

$$
\begin{aligned}
& M_{0}|u-v|^{1+2 \beta} \geq \int_{u}^{v} \int_{u}^{v} \int_{u}^{v} \frac{d x d y d z}{R^{2}(\Gamma(x), \Gamma(y), \Gamma(z))} \\
& \geq \int_{X} \int_{Y_{x}} \int_{Z_{x, y}} \frac{d z d y d x}{R^{2}(\Gamma(x), \Gamma(y), \Gamma(z))} \\
& \geq \frac{c_{1}}{c_{2}^{2}} \int_{X} \int_{Y_{x}}|\Gamma(x)-\Gamma(y)|^{2 \beta-1} d y d x \\
& \underset{\substack{(6.12),(6.13) \\
\geq}}{ } \frac{c_{1}}{4 c_{2}^{2}}|u-v|^{-1} \int_{X} \int_{Y_{x}}|x-y|^{2 \beta} d y d x .
\end{aligned}
$$

To deal with the integral over $Y_{x}$, we split it as

$$
\int_{Y_{x}}|x-y|^{2 \beta} d y=\int_{Y_{x} \cap\{y>x\}}(y-x)^{2 \beta} d y+\int_{Y_{x} \cap\{y \leq x\}}|y-x|^{2 \beta} d y
$$

and invoke the following simple fact for each of the two integrals.

If $g: \mathbb{R}_{+} \rightarrow \mathbb{R}_{+}$is monotone increasing and $Y \subset \mathbb{R}_{+}$is a bounded measurable set, then

$$
\int_{Y} g(y) d y \geq \int_{0}^{\mathscr{H}^{1}(Y)} g(t) d t .
$$

Therefore, applying the inequality $a^{2 \beta+1}+b^{2 \beta+1} \geq \frac{1}{8}(a+b)^{2 \beta+1}$ which is valid for $a, b \geq 0$ and $\beta \in(0,1]$, we obtain

$$
\int_{Y_{x}}|x-y|^{2 \beta} d y \geq \frac{1}{8} \cdot \frac{1}{2 \beta+1} \mathscr{H}^{1}\left(Y_{x}\right)^{2 \beta+1} \geq \frac{1}{24}\left(c_{1}|u-v|\right)^{2 \beta+1}
$$

for every $x \in X$. Inserting this into the earlier estimates (6.14), we check that

$$
M_{0}|u-v|^{1+2 \beta} \geq \frac{c_{1}}{4 c_{2}^{2}}|u-v|^{-1} \int_{X} \int_{Y_{X}}|x-y|^{2 \beta} d y d x \geq \frac{c_{1}^{4}}{100 c_{2}^{2}}|u-v|^{2 \beta} \mathscr{H}^{1}(X) .
$$


Hence,

$$
\begin{gathered}
\mathscr{H}^{1}(X) \leq \frac{100 c_{2}^{2} M_{0}}{c_{1}^{4}} \cdot|u-v| \\
\stackrel{(6.7)}{=} \frac{1}{4} c_{1}|u-v| .
\end{gathered}
$$

For the sake of geometric considerations later on, we also note that

$$
|\Gamma(x)-\Gamma(y)| \leq c_{2}|\Gamma(x)-\Gamma(y)|^{1-\beta} \quad \text { for all } x, y \in[u, v],
$$

since for $x, y \in[u, v]$ we have

$$
|\Gamma(x)-\Gamma(y)| \leq|x-y| \leq|u-v| \leq \delta_{1} \leq c_{2}^{1 / \beta} .
$$

By (6.15), there exists a point $x \in[u, v] \backslash X$ such that $|x-u| \leq c_{1}|u-v| \leq$ $\frac{1}{6 N}|u-v|$. We fix such a point $x$. By $(6.11), \mathscr{H}^{1}\left(Y_{x}\right)<c_{1}|u-v|$, since $x \notin X$. Thus we can select another point $y \in[u, v] \backslash Y_{x}$ such that $|y-v| \leq c_{1}|u-v| \leq \frac{1}{6 N}|u-v|$.

Now, set $r:=c_{2}|\Gamma(x)-\Gamma(y)|^{1-\beta}$ and let

$$
U:=U(\Gamma(x), \Gamma(y)), \quad V:=V(\Gamma(x), \Gamma(y) ; r), \quad \ell_{x, y}:=\ell(\Gamma(x), \Gamma(y) ; r) .
$$

We consider the following set of good parameters:

$$
S:=\left\{s \in(x, y): s \notin Z_{x, y}, s \notin Y_{x}, \Gamma(s) \in U\right\} .
$$

To estimate the difference $\left|\Gamma^{\prime}(x)-\Gamma^{\prime}(y)\right|$, we need to show first that the image $\Gamma(S)$ is contained in $\ell_{x, y}$ and that $S$ occupies a large portion of $[x, y]$.

Claim 6.3. We have $\Gamma(S) \subset V \cap U$.

Indeed, suppose there is a point $s \in S$ with $\Gamma(s) \in U \backslash V$. Then, the arc $\Gamma((x, y))$ passes through $\Gamma(s)$ and therefore

$$
\begin{aligned}
|x-y|=\mathscr{H}^{1}(\Gamma((x, y))) & \geq|\Gamma(x)-\Gamma(s)|+|\Gamma(s)-\Gamma(y)| \\
& \geq 2 r \\
& \stackrel{(6.16)}{\geq} 2|\Gamma(x)-\Gamma(y)| \\
& \stackrel{(6.8)}{\geq} 2 \lambda|x-y|>|x-y|,
\end{aligned}
$$

a contradiction. Thus, Claim 6.3 is established.

Claim 6.4. $\Gamma(S) \subset \ell_{x, y}$.

This follows directly from Claim 6.3 and the definition of $Z_{x, y}$.

Claim 6.5. We have

$$
\mathscr{H}^{1}(S) \geq|x-y|\left(\lambda-3 c_{1}\right) .
$$


Indeed, by definition of $S$,

$$
\begin{aligned}
\mathscr{H}^{1}(S) & \geq|\Gamma(x)-\Gamma(y)|-\mathscr{H}^{1}\left(Z_{x, y}\right)-\mathscr{H}^{1}\left(Y_{x}\right) \\
& >\lambda|x-y|-c_{1}|\Gamma(x)-\Gamma(y)|-c_{1}|u-v| \\
& \geq\left(\lambda-c_{1}\right)|x-y|-c_{1}|u-v| .
\end{aligned}
$$

(Note that the second inequality above is strict since $x \notin X$.) The choice of constants guarantees that $c_{1} \in\left(0, \frac{1}{N}\right)$. Therefore, $|x-y| \geq\left(1-\frac{2}{N}\right)|u-v|$, and $c_{1}|u-v| \leq 2 c_{1}|x-y|$, as $N>4$. Inequality (6.18) follows.

Set $\omega:=|\Gamma(x)-\Gamma(y)| / 2 N$. We now select two auxiliary good parameters $z_{1}, z_{2} \in S$.

Claim 6.6. There exist two points $z_{1}, z_{2} \in S$ such that

$$
\begin{gathered}
\Gamma\left(z_{1}\right) \in B_{\omega}(\Gamma(x)) \cap \ell_{x, y}, \quad \Gamma\left(z_{2}\right) \in B_{\omega}(\Gamma(y)) \cap \ell_{x, y}, \\
\max \left(\left|x-z_{1}\right|,\left|y-z_{2}\right|\right) \leq \frac{|x-y|}{N} .
\end{gathered}
$$

Again, we argue by contradiction. Let $B:=B_{\omega}(\Gamma(x))$. Suppose that $\Gamma(S) \cap B$ is empty. Then, by Claim 6.4, we have $\Gamma(S) \subset \ell_{x, y} \backslash B$. Choose $u_{0} \in(x, y)$ such that such that $\Gamma\left(u_{0}\right) \in \partial B$ and $\Gamma\left(\left(x, u_{0}\right)\right) \subset B$. We then necessarily have $S \subset\left[u_{0}, y\right]$ and

$$
\left|x-u_{0}\right| \geq\left|\Gamma(x)-\Gamma\left(u_{0}\right)\right|=\omega=\frac{1}{2 N}|\Gamma(x)-\Gamma(y)| \geq \frac{\lambda}{2 N}|x-y| .
$$

Thus,

$$
\mathscr{H}^{1}(S) \leq\left|u_{0}-y\right| \leq\left(1-\frac{\lambda}{2 N}\right)|x-y| .
$$

However, this inequality contradicts the previous estimate (6.18) (one can check that the choice of $c_{1}$ and $\lambda$ guarantees $\left.\lambda-3 c_{1}>1-\lambda / 2 N\right)$. Hence, there exists a point $z_{1} \in S$ such that $\Gamma\left(z_{1}\right) \in B \cap \ell_{x, y}$. (Recall that $\Gamma(S) \subset \ell_{x, y}$ by Claim 6.4.)

Moreover, we have

$$
\lambda\left|x-z_{1}\right| \leq\left|\Gamma(x)-\Gamma\left(z_{1}\right)\right| \leq \omega=\frac{1}{2 N}|\Gamma(x)-\Gamma(y)| \leq \frac{|x-y|}{2 N} .
$$

Thus, $\left|x-z_{1}\right| \leq|x-y| / N$. To prove the existence of $z_{2} \in S$, we proceed analogously; Claim 6.6 follows.

We are now ready to estimate $\left|\Gamma^{\prime}(u)-\Gamma^{\prime}(v)\right|$. Let

$$
\begin{array}{rlrl}
S(\tau, \sigma):=\frac{\Gamma(\tau)-\Gamma(\sigma)}{|\Gamma(\tau)-\Gamma(\sigma)|}, & & \tau \neq \sigma \in S_{L}, \\
Q(\tau, \sigma):=\frac{\Gamma(\tau)-\Gamma(\sigma)}{\tau-\sigma} & \tau \neq \sigma \in S_{L} .
\end{array}
$$


Applying the triangle inequality, we write

$$
\begin{aligned}
\left|\Gamma^{\prime}(u)-\Gamma^{\prime}(v)\right| & \leq\left|\Gamma^{\prime}(u)-\Gamma^{\prime}(x)\right|+\left|\Gamma^{\prime}(y)-\Gamma^{\prime}(v)\right|+\left|\Gamma^{\prime}(x)-\Gamma^{\prime}(y)\right| \\
& \leq 2 \Phi\left(\frac{|u-v|}{N}\right)+\left|\Gamma^{\prime}(x)-\Gamma^{\prime}(y)\right| .
\end{aligned}
$$

Next,

$$
\begin{aligned}
\left|\Gamma^{\prime}(x)-\Gamma^{\prime}(y)\right| \leq & \left|\Gamma^{\prime}(x)-S\left(z_{1}, x\right)\right|+\left|S\left(z_{1}, x\right)-S\left(y, z_{2}\right)\right| \\
& +\left|S\left(y, z_{2}\right)-\Gamma^{\prime}(y)\right| .
\end{aligned}
$$

We estimate each term separately.

Approximation of $\Gamma^{\prime}(x)$ by secant $S\left(z_{1}, x\right)$. Here we just use the information that $\Gamma^{\prime} \in C^{\alpha}$ and $z_{1}$ is very close to $x$. We write

$\left|\Gamma^{\prime}(x)-S\left(z_{1}, x\right)\right| \leq\left|\Gamma^{\prime}(x)-Q\left(z_{1}, x\right)\right|+\left|Q\left(z_{1}, x\right)-S\left(z_{1}, x\right)\right| \leq 2\left|\Gamma^{\prime}(x)-Q\left(z_{1}, x\right)\right|$.

This holds since $S\left(z_{1}, x\right)$ is the projection of $Q\left(z_{1}, x\right)$ onto the unit sphere $\mathbb{S}^{2}$ and therefore the closest point to $Q\left(z_{1}, x\right)$ on $\mathbb{S}^{2}$, and $\Gamma^{\prime}(x)$ is just another point in $\mathbb{S}^{2}$. Thus, by the fundamental theorem of calculus,

$$
\begin{aligned}
\left|\Gamma^{\prime}(x)-S\left(z_{1}, x\right)\right| & \leq 2 f_{\left[x, z_{1}\right]}\left|\Gamma^{\prime}(x)-\Gamma^{\prime}(s)\right| d s \leq 2 \Phi\left(\left|x-z_{1}\right|\right) \\
& \leq 2 \Phi(|x-y| / N) \leq 2 \Phi(|u-v| / N)
\end{aligned}
$$

as $\left|x-z_{1}\right| \leq|x-y| / N<|u-v| / N$.

In a similar way, we obtain

$$
\left|\Gamma^{\prime}(y)-S\left(y, z_{2}\right)\right| \leq 2 \Phi(|u-v| / N) .
$$

The difference between two secants. Here we use the fact that $\Gamma\left(z_{i}\right) \in \ell_{x, y}$ for $i=1,2$. We apply the triangle inequality again to write

$$
\left|S\left(z_{1}, x\right)-S\left(y, z_{2}\right)\right| \leq\left|S\left(z_{1}, x\right)-S(y, x)\right|+\left|S(y, x)-S\left(y, z_{2}\right)\right|,
$$

and invoke Lemma 2.2 to estimate each term. This gives

$$
\begin{aligned}
\left|S\left(z_{1}, x\right)-S\left(y, z_{2}\right)\right| & \leq \frac{2}{c_{2}}|\Gamma(x)-\Gamma(y)|^{\beta} \\
& \leq c_{0}|x-y|^{\beta}
\end{aligned}
$$

where $c_{0}=2 / c_{2}=\operatorname{const}(\alpha) \cdot M_{0}^{1 / 2}$. Combining the estimates $(6.25),(6.26)$ and (6.27) with (6.24), we finally obtain

$$
\left|\Gamma^{\prime}(x)-\Gamma^{\prime}(y)\right| \leq 4 \Phi(|u-v| / N)+c_{0}|x-y|^{\beta} .
$$

The conclusion of Lemma 6.1 follows immediately from (6.23) and (6.28). 


\subsection{Conclusion: proofs of the main results}

In this subsection, we simply collect all facts that have been proved earlier and show how to assemble them to obtain full proofs of Theorems 1.1-1.4.

Proof of Theorem 1.3 (ii). Since $\Gamma$ is a local homeomorphism, we can use Theorem 3.8 to conclude that $\Gamma$ is a $k$-fold covering and to reparametrize $\gamma$. The new (injective) parametrization $\Gamma_{1}: S_{L / k} \rightarrow \mathbb{R}^{3}$ is of class $C^{1, \alpha}$ for $\alpha=2 \beta /(6-4 \beta) \leq \beta$ (cf. Section 2). Thus, Lemma 6.1 can be applied, and we obtain $\Gamma_{1} \in C^{1, \beta}$. This regularity statement carries over to the original $k$-fold covering $\Gamma$, as $\Gamma$ is periodic and the choice of 0 on the original circle $S_{L}$ is arbitrary.

Proof of Theorem 1.3 (i). Note that by definition of the intermediate radius $\varrho[\gamma]$ we have

$$
\varrho[\gamma](s, t) \leq R(\Gamma(s), \Gamma(t), \Gamma(\sigma)) \quad \text { for all } \sigma \in S_{L} .
$$

Thus, for any triple of balls $B_{i}=B_{r}\left(\tau_{i}\right) \subset S_{L}, i=1,2,3$, we obtain

$$
\int_{B_{1}} \int_{B_{2}} \frac{d s d t}{\varrho[\gamma](s, t)^{2}} \geq \int_{B_{1}} \int_{B_{2}} \frac{d s d t}{R(\Gamma(s), \Gamma(t), \Gamma(\sigma))^{2}} \quad \text { for all } \sigma \in B_{3},
$$

and integration over $B_{3}$ yields

$$
2 r \int_{B_{1}} \int_{B_{2}} \frac{d s d t}{\varrho[\gamma](s, t)^{2}} \geq \int_{B_{1}} \int_{B_{2}} \int_{B_{3}} \frac{d s d t d \sigma}{R(\Gamma(s), \Gamma(t), \Gamma(\sigma))^{2}} .
$$

It is now clear that (1.8) implies the decay assumption (1.9); one just has to replace $M_{0}$ by $2 M_{0}$ in (1.9). Moreover, see Section 3.4, we know that (1.8) guarantees that $\Gamma$ is injective. Thus, Theorem 1.3 (ii) ascertains that $\Gamma \in C^{1, \beta}$.

Proof of Theorem 1.4. By Theorem 3.1, we know that $\Gamma\left(S_{L}\right)$ is a one-dimensional topological submanifold in $\mathbb{R}^{3}$. Besides that, $\gamma$ is a continuous rectifiable curve. Thus, we can reparametrize $\Gamma\left(S_{L}\right)$; the new arclength parametrization

$$
\Gamma_{1}:\left[0, L_{1}\right] \stackrel{\text { onto }}{\longrightarrow} \Gamma\left(S_{L}\right) \subset \mathbb{R}^{3}
$$

will be injective. Moreover, we have $\Gamma_{1}^{\prime} \in C^{\alpha}$ by the results of Section 2, as (1.9) holds for $\Gamma_{1}$. Therefore, we may apply Lemma 6.1 to obtain $\Gamma_{1} \in C^{1, \beta}$.

Proofs of Theorem 1.1 and 1.2. Assume first that $p>2$ and set $\beta=1-\frac{2}{p}$. For any couple of balls $B_{i}=B_{r}\left(\tau_{i}\right) \subset S_{L}, i=1,2$, the Hölder inequality gives

$$
\int_{B_{1}} \int_{B_{2}} \frac{d s d t}{\varrho[\gamma](s, t)^{2}} \leq(2 r)^{2 \beta}\left(\int_{B_{1}} \int_{B_{2}} \frac{d s d t}{\varrho[\gamma](s, t)^{p}}\right)^{2 / p} \leq(2 r)^{2 \beta} \mathscr{I}_{p}(\gamma)^{2 / p} .
$$

Thus, (1.5) implies (1.8) with $M_{0}:=2^{2 \beta} \mathscr{I}_{p}(\gamma)^{2 / p}$, and Theorem 1.1 follows from Proposition 3.10 and Theorem 1.3 (i).

Similarly, we have already noted earlier that (1.6) implies (1.9) for $\beta=1-\frac{3}{p}$ and $M_{0} \lesssim \mathscr{M}_{p}(\gamma)^{2 / p}$. Thus, Theorem 1.2 follows from Theorem 1.3 (ii). 


\section{Variants of the main results}

It turns out that all the main results of the paper do hold under slightly weaker assumptions. Namely, it suffices to assume that the integrals of $1 / R$ - and not of $1 / R^{2}$ - decay to zero with an appropriate speed. This observation follows from careful analysis of the proofs in Sections 5 and 6 . Below, we briefly indicate the most important changes, leaving all other details to the interested reader.

Remark 7.1. It is an exercise to see that in the proof of Lemma 6.1 one might replace assumption (6.2) by a weaker one,

$$
\sup _{\substack{0<r \leq r_{0} \\ \tau \in S_{L}}} r^{-(2+\beta)} \int_{B_{r}(\tau)} \int_{B_{r}(\tau)} \int_{B_{r}(\tau)} \frac{d x d y d z}{R(\Gamma(x), \Gamma(y), \Gamma(z))} \leq K_{0} .
$$

In fact, it is possible to work with the same sets (6.9)-(6.11) of 'bad' parameters, leaving $\lambda, c_{1}, \delta_{2}$ and $\delta_{1}$ in (6.6), (6.7) unchanged, and replacing the old $c_{2}$ defined in (6.7) by a new constant $c_{2}:=2^{-7} K_{0}^{-1} c_{1}^{4}$. After minor technical adjustments in the estimate (6.14), we can replace the old estimate of the set $X$ of 'bad' parameters, i.e. (6.15), by

$$
\mathscr{H}^{1}(X) \leq \frac{2^{5} c_{2} K_{0}}{c_{1}^{3}}|u-v|=\frac{1}{4} c_{1}|u-v| .
$$

From that point on, the proof of Lemma 6.1 goes without changes.

Remark 7.2. One can also check that it is possible to replace (2.8) in the Uniform cone flatness theorem (and therefore also (1.9) in Assumption (ii) of Theorem 1.3) by an analogous assumption,

$$
\int_{B_{r}\left(\tau_{1}\right)} \int_{B_{r}\left(\tau_{2}\right)} \int_{B_{r}\left(\tau_{3}\right)} \frac{d s d t d \sigma}{R(\Gamma(s), \Gamma(t), \Gamma(\sigma))} \leq K_{0} r^{2+\beta}
$$

for all $0<r \leq r_{0}$ and all $\tau_{1}, \tau_{2}, \tau_{3} \in S_{L}$.

Again, the proof is very similar to the proof of Theorem 2.3. The whole idea and a substantial (geometric) part of the proof stays unchanged; adjusting minor technical details (i.e., the constants and exponents) in the computations (5.18)(5.21) we obtain the following:

Theorem 7.3 (Uniform cone flatness III). Assume that there are constants $\beta \in$ $(0,1], r_{0}>0$, and $M_{0} \geq 0$, such that $\Gamma$ satisfies (7.2) for all $0<r \leq r_{0}$ and all $\tau_{1}, \tau_{2}, \tau_{3} \in S_{L}$. Then there exists a constant $\tilde{c}=\tilde{c}(\beta)>1$ depending only on $\beta$ such that if two numbers, $\varepsilon \in(0, \pi / 2)$ and $\eta>0$, satisfy the relation

$$
\varepsilon^{3-2 \beta} \geq \tilde{c}(\beta) K_{0} \eta^{\beta} \text { and } \eta \leq \min \left\{\frac{1}{2} \operatorname{diam} \Gamma\left(S_{L}\right), r_{0}\right\},
$$


then for every $s, t \in S_{L}$ such that $|\Gamma(s)-\Gamma(t)|=\eta$ we have

$$
\Gamma\left(S_{L}\right) \cap B_{2 \eta}(\Gamma(s)) \subset C_{\varepsilon}(\Gamma(s) ; \Gamma(t)) .
$$

It is clear that this result might serve as a replacement of Theorem 2.3 in numerous proofs that were presented in Sections 2 and 3. This would require a few more changes of notation; in particular, the definitions (2.2) and (2.3) of the diamondlike sets $D(a, b)$ and $D_{U}(a, b)$ are based on (2.1) and were designed to work well in various applications of Theorem 2.3, but this is a minor point. However, Proposition 2.7 and Corollary 2.8 (uniform local injectivity) do hold when one replaces the decay assumption (2.8) by (7.2); one just has to adjust the constant $\delta_{0}$. Similarly, a counterpart of Theorem 2.10 holds:

If the arclength parametrization $\Gamma: S_{L} \rightarrow \mathbb{R}^{3}$ of a curve $\gamma: \mathbb{S}^{1} \rightarrow \mathbb{R}^{3}$ satisfies (7.2) for all $0<r \leq r_{0}$ and all $\tau_{1}, \tau_{2}, \tau_{3} \in S_{L}$, and $\Gamma$ is a local homeomorphism, then $\Gamma^{\prime}$ exists everywhere on $S_{L}$, and there is a constant $C_{\Gamma}$ depending on diam $\gamma, r_{0}, \beta$, and $K_{0}$, such that

$$
\left|\Gamma^{\prime}(s)-\Gamma^{\prime}(t)\right| \leq C_{\Gamma}|s-t|^{\frac{\beta}{3-2 \beta}} \quad \text { for all } s, t \in S_{L} .
$$

\section{References}

[1] T. Ashton, J. Cantarella, M. Piatek and E. Rawdon, Self-contact sets for 50 tightly knotted and linked tubes, arXiv:math.DG/0508248 v1 (2005).

[2] J. R. BAnavar, O. Gonzalez, J. H. Maddocks and A. Maritan, Self-interactions of strands and sheets, J. Stat. Phys. 110 (2003), 35-50.

[3] L. M. Blumenthal and K. Menger, "Studies in Geometry", Freeman and co., San Francisco, CA, 1970.

[4] J. Cantarella, J. H. G. Fu, R. B. Kusner, J. M. Sullivan and N. C. Wrinkle, Criticality for the Gehring link problem, Geom. Topol. 10 (2006), 2055-2116.

[5] J. Cantarella, R. B. Kusner and J. M. Sullivan, On the minimum ropelength of knots and links, Invent. Math. 150 (2002), 257-286.

[6] J. Cantarella, M. Piatek and E. Rawdon, Visualizing the tightening of knots, In: "VIS'05: Proc. of the 16th IEEE Visualization 2005", IEEE Computer Society, Washington, DC, 2005, 575-582.

[7] M. Carlen, B. Laurie, J. H. Maddocks and J. Smutny, Biarcs, global radius of curvature, and the computation of ideal knot shapes, In: "Physical and Numerical Models in Knot Theory", J. A. Calvo, K. C. Millett, E. J. Rawdon, A. Stasiak (eds.) Ser. on Knots and Everything 36, World Scientific, Singapore, 2005, 75-108.

[8] R. H. Crowell and R. H. Fox, "Introduction to Knot Theory", Springer, New York, 1977. (Reprint of the 1963 original, Graduate Texts in Mathematics, Vol. 57.)

[9] G. DAVID and S. Semmes, "Singular Integrals and Rectifiable Sets in $\mathbb{R}^{n}$ : Au-delà des graphes lipschitziens", Astériques 193, Soc. Mathématique France, Montrouge, 1991.

[10] M. H. Freedman, Z.-X. HE and Z. WANG, Möbius energy of knots and unknots, Ann. of Math. 139 (1994), 1-50.

[11] H. GERLACH and J. H. MADDOCKS, Existence of ideal knots in $\mathbb{S}^{3}$, in preparation. 
[12] H. GeRlach and H. VON DER MOSEL, What are the longest ropes on the unit sphere? Preprint Nr. 32, Institut für Mathematik, RWTH Aachen University (2009); see http://www.instmath.rwth-aachen.de/ heiko/veroeffentlichungen/longest_ropes.pdf.

[13] O. GonZalez and R. DE LA Llave, Existence of ideal knots, J. Knot Theory Ramifications 12 (2003), 123-133.

[14] O. GonZALEZ and J. H. MADDOCKS, Global curvature, thickness, and the ideal shape of knots, Proc. Natl. Acad. Sci. USA 96 (1999), 4769-4773.

[15] O. Gonzalez, J. H. Maddocks, F. Schuricht and H. von DeR Mosel, Global curvature and self-contact of nonlinearly elastic curves and rods, Calc. Var. Partial Differential Equations 14 (2002), 29-68.

[16] I. HahlomaA, Menger curvature and Lipschitz parametrizations in metric spaces, Fund. Math. 185 (2005), 143-169.

[17] I. HAHLOMAA, Curvature integral and Lipschitz parametrization in 1-regular metric spaces, Ann. Acad. Sci. Fenn. Math. 32 (2007), 99-123.

[18] J. C. LÉGER, Menger curvature and rectifiability, Ann. of Math. 149 (1999), 831-869.

[19] G. LERMAN and J. T. Whitehouse, High-dimensional Menger-type curvatures - Part I: Geometric multipoles and multiscale inequalities, arXiv:0805.1425v1 (2008), to appear in Rev. Mat. Iberoamericana.

[20] G. LERMAN and J. T. Whitehouse, High-dimensional Menger-type curvatures - Part II: $d$-Separation and a menagerie of curvatures, Constr. Approx. 30 (2009), 325-360.

[21] Y. Lin and P. Mattila, Menger curvature and $C^{1}$-regularity of fractals, Proc. Amer. Math. Soc. 129 (2000), 1755-1762.

[22] P. Mattila, Rectifiability, analytic capacity, and singular integrals, In: "Proc. ICM", Vol. II, Berlin 1998, Doc. Math. 1998, Extra Vol. II, 657-664 (electronic).

[23] P. MatTiLA, Search for geometric criteria for removable sets of bounded analytic functions, Cubo 6 (2004), 113-132.

[24] M. Melnikov, Analytic capacity: discrete approach and curvature of measure, Sb. Mat. 186 (1995), 827-846.

[25] M. Melnikov and J. Verdera, A geometric proof of the $L^{2}$ boundedness of the Cauchy integral on Lipschitz curves, Int. Math. Res. Not. 7 (1995), 325-331.

[26] K. Menger, Untersuchungen über allgemeine Metrik. Vierte Untersuchung, Zur Metrik der Kurven, Math. Ann. 103 (1930), 466-501.

[27] H. PAjot, "Analytic Capacity, Rectifiability, Menger Curvature and the Cauchy Integral", Springer Lecture Notes, Vol. 1799, Springer Berlin, Heidelberg, New York, 2002.

[28] R. Schul, Ahlfors-regular curves in metric spaces, Ann. Acad. Sci. Fenn. Math. 32 (2007), 437-460.

[29] F. SCHURICHT and H. VON DER Mosel, Global curvature for rectifiable loops, Math. Z. 243 (2003), 37-77.

[30] F. SCHURICHT and H. VON DER MOSEL, Euler-Lagrange equations for nonlinearly elastic rods with self-contact, Arch. Ration. Mech. Anal. 168 (2003), 35-82.

[31] F. SCHURICHT and H. VON DER Mosel, Characterization of ideal knots, Calc. Var. Partial Differential Equations 19 (2004), 281-305.

[32] P. Strzelecki, M. SzumańsKa and H. VON Der Mosel, a geometric curvature double integral of Menger type for space curves Ann. Acad. Sci. Fenn. Math. 34 (2009), 195-214.

[33] P. STRZELECKI and H. VON DER MOSEL, On a mathematical model for thick surfaces, In: "Physical and Numerical Models in Knot Theory", J. A. Calvo, K. C. Millett, E. J. Rawdon, A. Stasiak (eds.), Ser. on Knots and Everything 36, World Scientific, Singapore, 2005, 547564.

[34] P. Strzelecki and H. VON Der Mosel, Global curvature for surfaces and area minimization under a thickness constraint, Calc. Var. Partial Differential Equations 25 (2006), 431-467.

[35] P. STRZelecKI and H. VON Der Mosel, On rectifiable curves with $L^{p}$-bounds on global curvature: Self-avoidance, regularity, and minimizing knots, Math. Z. 257 (2007), 107-130. 
[36] P. STRZelecki and H. VON DER MOSEl, Integral Menger curvature for surfaces, arXiv:math.CA/0911.2095 v2 (2009).

[37] J. VERDERA, The $L^{2}$ boundedness of the Cauchy integral and Menger curvature, In: "Harmonic Analysis and Boundary Value Problems", Contemp. Math. 277, AMS, Providence, RI, 2001, 139-158.

Instytut Matematyki

Uniwersytet Warszawski

ul. Banacha 2

PL-02-097 Warsaw, Poland

pawelst@mimuw.edu.pl

Instytut Matematyczny

Polskiej Akademii Nauk

ul. Śniadeckich 8

PL-00-950 Warsaw, Poland

m.szumanska@gmail.com

Institut für Mathematik

RWTH Aachen University

Templergraben 55

D-52062 Aachen, Germany

heiko@instmath.rwth-aachen.de 